\title{
Telehealth and Autism Prior to and in the Age of COVID-19: A Systematic and Critical Review of the Last Decade
}

\author{
Kimberly S. Ellison ${ }^{1} \cdot$ Jerrica Guidry ${ }^{1} \cdot$ Paige Picou $^{1} \cdot$ Paige Adenuga $^{1} \cdot$ Thompson E. Davis III $^{1}$ (])
}

Accepted: 30 May 2021 / Published online: 10 June 2021

(c) The Author(s), under exclusive licence to Springer Science+Business Media, LLC, part of Springer Nature 2021

\begin{abstract}
There has been growing interest in the use of telehealth; however, the COVID-19 pandemic and the subsequent isolation and restrictions placed on in-person services have fast-tracked implementation needs for these services. Individuals with autism spectrum disorder (ASD) have been particularly affected due to the often-intensive service needs required by this population. As a result, the aim of this review was to examine the evidence base, methodology, and outcomes of studies that have used telehealth for assessment and/or intervention with children and adolescents with ASD as well as their families over the last decade. Further, the goal is to highlight the advances in telehealth and its use with this special population. A systematic search of the literature was undertaken, with 55 studies meeting inclusion criteria and quality analysis. Specified details were extracted from each article, including participant characteristics, technology, measures, methodology/study design, and clinical and implementation outcomes. Services provided via telehealth included diagnostic assessments, preference assessments, early intervention, applied behavior analysis (ABA), functional assessment and functional communication training, and parent training. Findings, although still emerging, encouragingly suggested that services via telehealth were equivalent or better to services face-to-face. Results support the benefits to using telehealth with individuals with ASD. Future research should continue to explore the feasibility of both assessments and interventions via telehealth with those having ASD to make access to assessment services and interventions more feasible for families, while acknowledging the digital divide it could create.
\end{abstract}

Keywords Autism Spectrum Disorder $\cdot$ ASD $\cdot$ Autism $\cdot$ Telehealth $\cdot$ Assessment $\cdot$ Intervention

\section{Introduction}

"Telehealth" is an all-encompassing term for the use of various modes of technology to provide medical and mental health care services in place or in addition to in-person methods (American Psychological Association, 2013). Services can be implemented via synchronous or asynchronous modalities, such as telephone calls, video-teleconferencing, email electronic applications, or video and audio recordings (American Psychiatric Association, 2013). The use of telehealth as a tool for implementing intervention and assessment services has grown recently particularly with the impacts of COVID-19 on in-person access to services. As technology has improved, the implementation of services

Thompson E. Davis III

ted@1su.edu

1 Department of Psychology, Louisiana State University, 236 Audubon Hall, Baton Rouge, LA 70803, USA via telehealth has been found to be cost-effective and can be delivered across vast geographic regions which would otherwise prevent access to care (Baweja et al., 2021; Shulver et al., 2016). Additionally, the further integration of technology in clinical practice has become more widely accepted due to increased convenience, decreased stigma, improved patient outcomes, and reduced expenses (Luxton et al., 2016). As telehealth evolves, improves, and gains further acceptance, clinicians have also begun to explore the implementation of telehealth psychological and behavioral services to individuals with autism spectrum disorder (ASD).

\section{Telehealth Applications for Autism Spectrum Disorder}

Autism spectrum disorder (ASD) is a neurodevelopmental disorder characterized by deficits in social communication and interactions and restricted, repetitive behaviors, interests, or activities (American Psychiatric Association, 2013). 
As ASD is a lifelong disorder with significant and cascading developmental implications, early identification and intervention have been found to be critical (Hyman et al., 2020). However, gaining access to intervention programs throughout the community is often a challenge for families who live in rural or remote areas, have limited or no transportation, are of lower socioeconomic status or experience other logistical difficulties (Sutherland et al., 2019). For example, children who are at or below the poverty line or live in rural areas are diagnosed with ASD much later than those children who are of high socioeconomic status or live in more urban areas (Antezana et al., 2017), which further delays their access to needed services. This disparity was likely even further impacted by the effects of the COVID-19 pandemic, where individuals who are below the poverty line are more at risk for experiencing health disparities and are advised to remain at home (Dahiya et al., 2020). Furthermore, many children with ASD had disruption to their services due to the COVID-19 pandemic, which was not only exacerbated by the state-wide mandatory shutdowns across the country but was also impacted by the decrease in staffing at these service providers (Eshraghi, 2020). Telehealth services, if effective and appropriate, may be able to address some of these concerns, as well as have the potential benefit of not disrupting a child with ASD's routine and daily schedule to the same degree with additional travel, time-lost, etc. It may also benefit the families as telehealth services have been found to be more cost-effective than in-person services (Camden \& Silva, 2021). While the effects of telehealth for children with ASD should not be assumed to be equivalent to those seen with in-person services or those seen in neurotypical individuals using the medium for other concerns, it remains to be seen if a consistent body of literature has begun to accrue to suggest telehealth may or may not be a viable option in this population. Despite the increased need for access to mental health services, the literature regarding the use of telehealth for assessment and intervention services with children with behavioral needs, such as ASD, is limited.

The most recent systematic review of research focusing on ASD and the use of telehealth was conducted by Sutherland et al. (2019). Sutherland et al. (2019), sought to review articles to inform the speech-language pathology field, and found only 14 studies that met their inclusion criteria: the inclusion of at least one person with ASD, implementation of a telehealth system for the purpose of an intervention or assessment, the use of a design that allows for experimental control or comparison conditions, measurement of factors associated with telehealth implementation (e.g., child outcomes, feasibility, parent outcomes), and published in a peer-reviewed journal. There were 284 participants involved in the 14 studies with an age range of 19 months to adulthood. The services included in those studies consisted of diagnostic assessment services, early interventions, anxiety interventions, functional behavior assessment and communication training, web-based education and consultation, and language interventions. Their study sought to extend the findings in the previous review by Boisvert et al. (2010) where eight studies were included in their telehealth review and concluded that telehealth is a promising mode to treatment for individuals with ASD. Overall, the findings of the Sutherland et al. (2019) review supported the implementation of telehealth services, highlighting an improvement in fidelity, reduction of behavior problems, an increase in parent satisfaction, and an increase in program acceptability. The studies were a mixture of repeated measures designs, pre- and post-intervention studies, randomized controlled trials, mixed-methods, multiple-baseline designs, case studies, and observational data (Sutherland et al., 2019).

With advances in technology, the literature using telehealth has grown significantly. Since the last systematic review in 2018, a search using PsycINFO with the terms "telehealth OR telepractice" yielded 1,429 articles. Based on this sharp increase, and due to the imminent and ongoing impact of the COVID-19 pandemic since the last review, and the increasingly expansive improvements in telehealth platforms and technology, there is a need for a current update and review of the literature on the use of mental health services with the ASD population to understand the utility and efficacy of this service modality for the "new normal" (e.g., ongoing social distancing, the wearing of masks, recurring and ever-changing restrictions on in-person gatherings and activities, etc.). The aim of this review was to provide an overview of the literature regarding telehealth for children and adolescents with ASD over the last decade, with regards to the type, recipients, and outcomes of the services and provide a recent evidence base upon which professionals and researchers alike might base ongoing and future services and research.

\section{Method}

The Preferred Reporting Items for Systematic Reviews and Meta-Analyses (PRISMA) was used to guide the systematic review process (see Fig. 1) based on its use in the previous review (Sutherland et al., 2019). A systematic search of the literature was conducted using the Medline, PsycINFO, ERIC, and CINAHL databases. These databases were selected based on the previous review by Boisvert et al. (2010) and Sutherland et al. (2019). Titles and abstracts were searched using key words to describe telehealth and ASD ("telehealth" OR "telemedicine" OR "telepractice" OR "telecare" AND "Autis"” allowing searches for Autism, Autistic, Autisms, etc.). Similar to the previous reviews, the search was limited to English and only articles from peer-reviewed journals were included. No eligible articles were found prior 


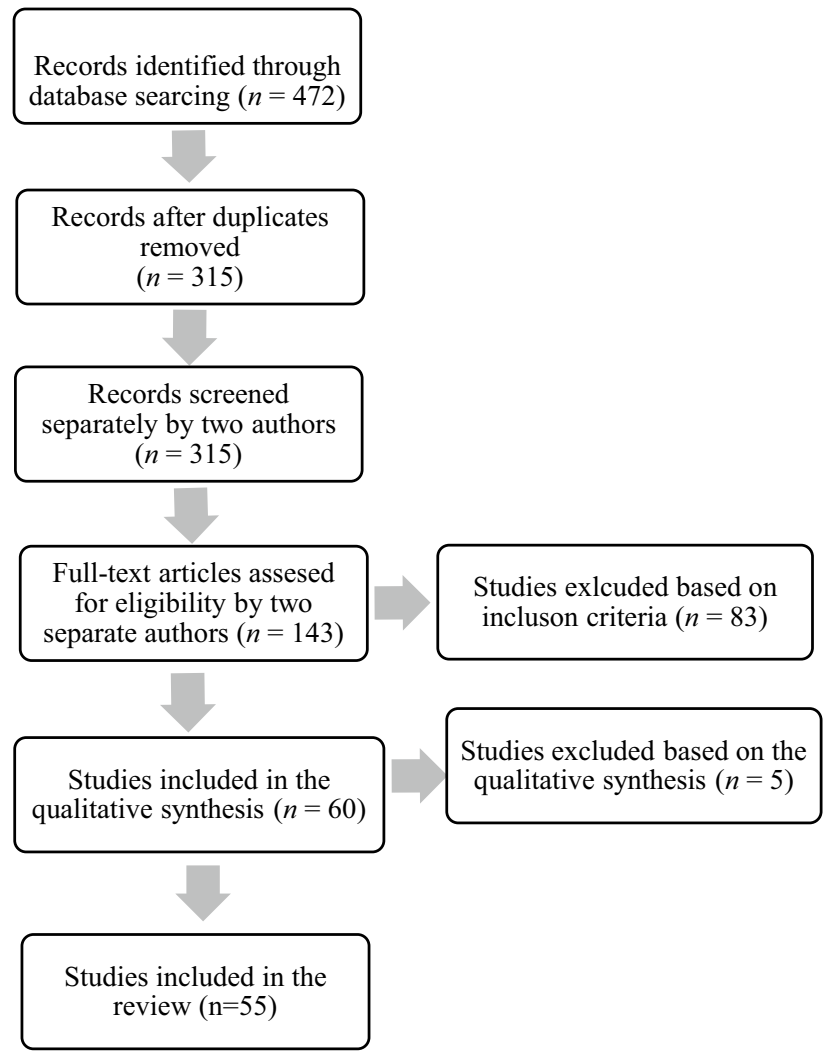

Fig. 1 PRISMA summary of paper screening process

to 2010 that were not included in the previous reviews (Boisvert et al., 2010; Sutherland et al., 2019). Based on the previous findings, this review included articles between December 2010 and March 2021 as a review of the last decade.

A total of 472 articles that included both the telehealth and autism search terms were found across the four databases. After duplicates were removed, a total of 315 remained. Titles and abstracts were screened separately by the first and second authors based on the predetermined inclusion criteria: (a) inclusion of one individual with autism or parent of a person with autism; (b) implementation of a telehealth system for the purpose of assessment or intervention; (c) the use of a design that allows for experimental control (e.g., intervention studies) or comparison condition (e.g., diagnostic studies); (d) measurement of factors associated with implementation (e.g., outcomes, feasibility, acceptability); and (e) published in a peer-review journal. Synchronous (e.g., real time consultation) and asynchronous (e.g., images, videos, applications) modalities were considered as telehealth services. Web-based materials used to train parent, teacher, or clinicians without any consultation were not included. General review articles and articles using software such as virtual reality or wearable sensors were excluded per previous reviews (Boisvert et al., 2010; Sutherland et al., 2019). After screening the 315 papers based on title and abstract, 143 articles remained. These articles were independently read in full by the first and second authors to determine eligibility. The two reviewers then discussed the articles to resolve any disagreements about inclusion of a study. Following the review, 60 papers remained that met all inclusion criteria.

A quality review of the articles that met the inclusion criteria was conducted using the Scientific Merit Rating Scales (SMRS; National Autism Center, 2015). The review included the process of rating the studies on five separate criteria for experimental rigor, including research design, measurement of dependent and independent variables, participant ascertainment, and generalization. The scientific merit score was obtained by combining the ratings of each criterion. Studies that met a score of 3,4 , or 5 indicated that scientific rigor had been utilized and firm conclusions can be drawn, while a score of 2 indicated initial evidence of intervention effects but more scientific rigor should be utilized to confirm these effects. Lastly, a score of 1 or 0 indicated that insufficient scientific rigor was applied to the studies. The studies were split between the second, third, and fourth authors to be rated based on the five criteria, while the first author independently rated all included studies. The ratings were then compared to the first author's ratings to ensure reliability. Articles were discussed if the absolute value of any of the individual variables and/or overall SMRS score was equal to one or greater; any discrepancies were discussed and resolved. After the ratings were completed, five studies were excluded from the review due to receiving a SMRS score of 1.9 or lower, which indicated a lack of scientific rigor. A total of 55 papers were included in the review as a result of these processes.

\section{Results}

All 55 papers reviewed were published between January 2010 and March 2021, across a range of disciplines. The first section of this review examines articles that emphasized the use of telehealth for either the assessment of ASD or other common assessments used to inform treatment of individuals with ASD (i.e., functional assessment, speech and language assessment, preference assessments). Details of the papers included in the assessment section of this review are summarized in Table 1. The next section outlines the different interventions utilized via telehealth for individuals with ASD. Details of the papers included in the intervention section of this review are summarized in Table 2. Within both the assessment and intervention sections, studies included in this review are organized by the telehealth participant in the following order: the individual with ASD him or herself, parents of individuals with ASD, and other interventionists/staff/teachers of individuals with ASD. The intervention 


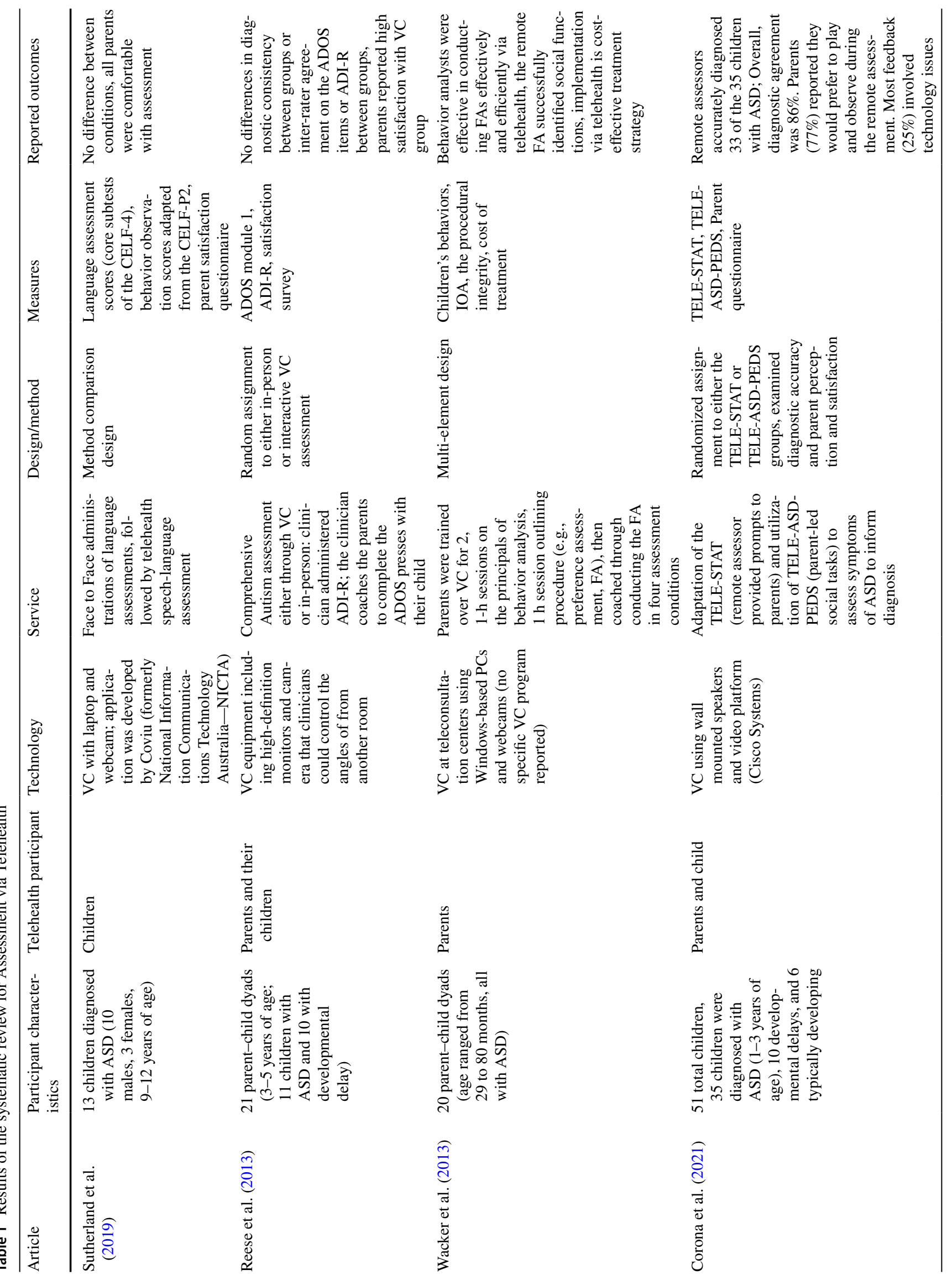




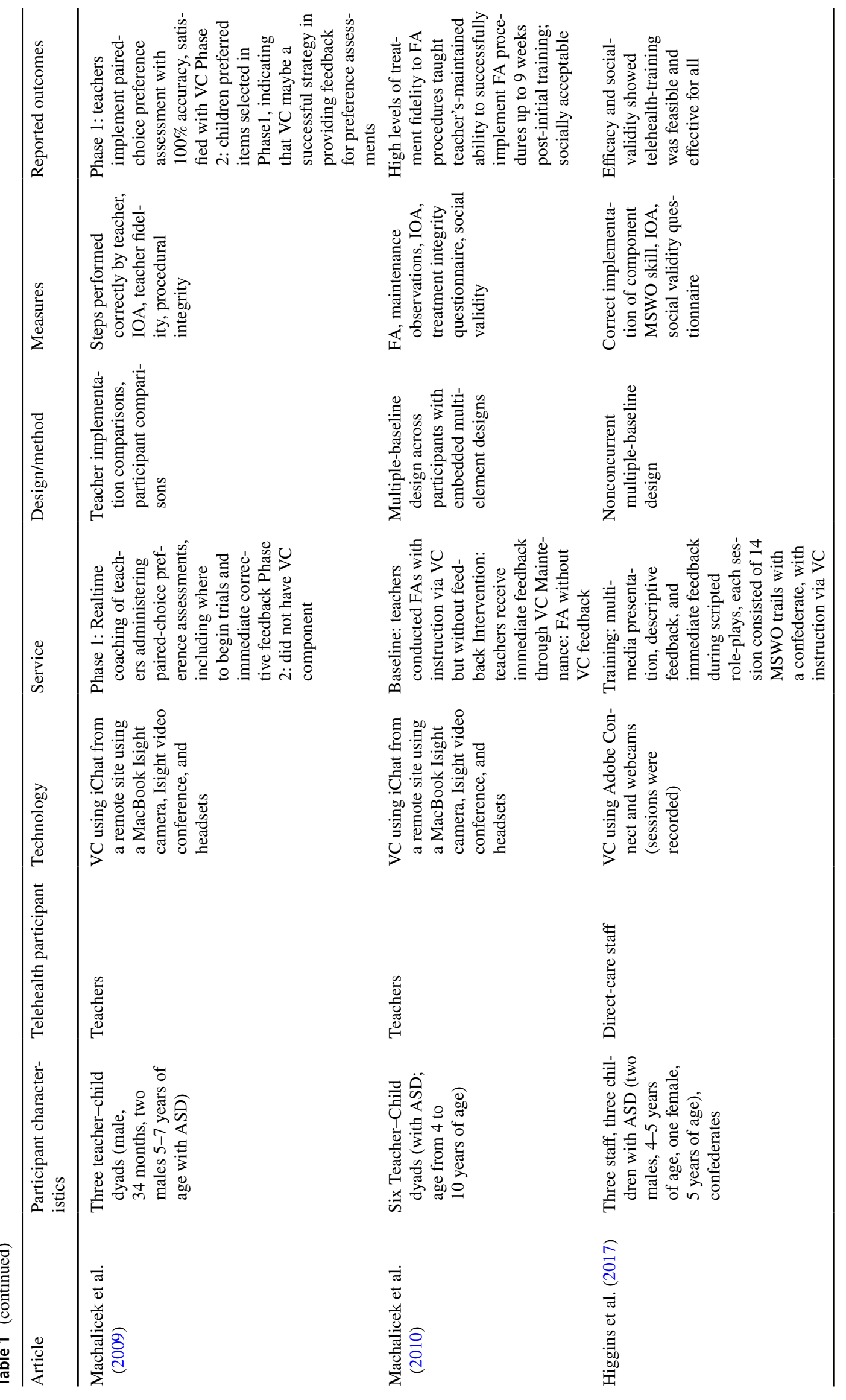




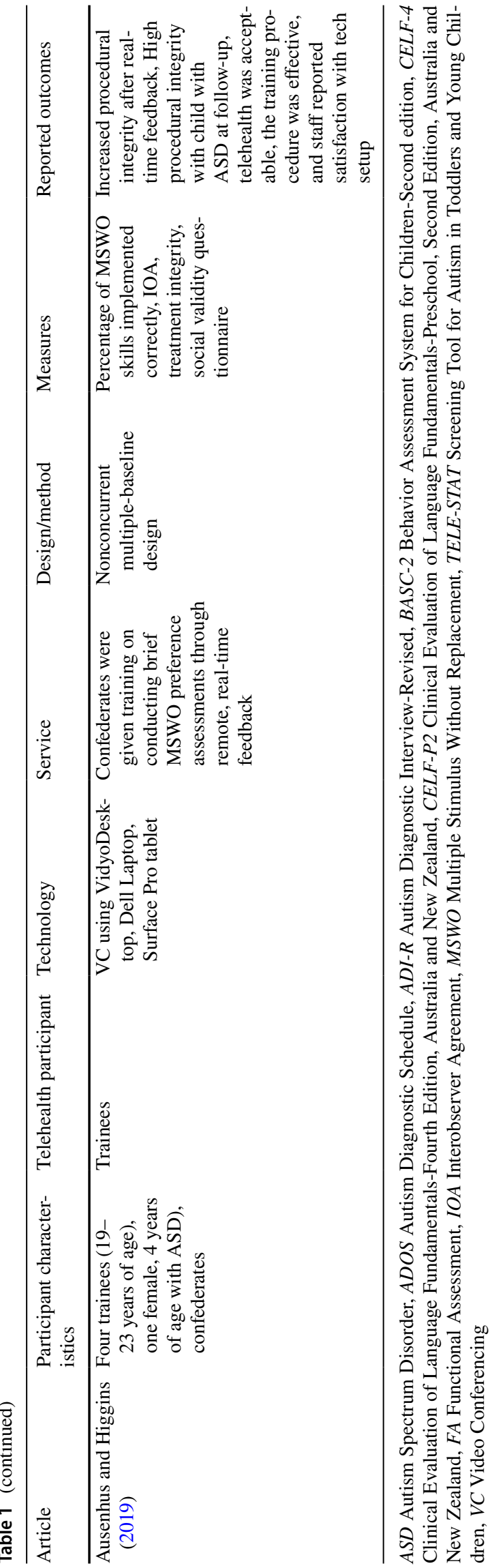

section also includes two studies that feature the telehealth participant as being a parent and a teacher together. Lastly, limitations and future directions for the use of telehealth with individuals with ASD are discussed.

\section{Assessment}

A total of eight studies implemented assessment procedures via telehealth for individuals with ASD. All of these studies used video conferencing (VC) to deliver the assessment procedures and utilized a variety of different VC systems (e.g., Cisco Systems, Isight via MacBooks, Adobe Connect, VidyoDesktop) (Ausenhus \& Higgins, 2019; Corona et al., 2021; Higgins et al., 2017; Machalicek et al., 2009, 2010; Reese et al., 2013; Wacker et al., 2013). One study, used an application, Coviu, that was created for VC (Sutherland et al., 2019).

\section{Telehealth Participant: Children with ASD}

Only one study utilized VC to administer four subtests of a speech and language assessment (Clinical Evaluation of Language Fundamentals, 4th Edition) remotely by a speechlanguage pathologist to children with ASD; this telehealth procedure was compared to in-person administrations of the same four subtests. The assessment scores were reportedly high in agreement between in-person and telehealth implementation, but no differences between the procedures were found (Sutherland et al., 2019). Parents' satisfaction with the telehealth assessment was high; they also indicated that their children felt either "somewhat or definitely" comfortable with the procedures as well (Sutherland et al., 2019).

\section{Telehealth Participant: Parent of Child with ASD}

Two studies utilized the parents of individuals with ASD to implement diagnostic autism assessments. One study (Reese et al., 2013), randomly assigned participants to either the in-person administration group or VC administration group. Both groups were administered the Autism Diagnostic Interview-Revised (Rutter et al., 2003). Video conferencing was utilized to coach parents in implementing modified Autism Diagnostic Observation Schedule (Lord et al., 2002)-Module 1 activities and presses with their children compared to an in-person autism assessment utilizing these same presses. No difference between diagnostic consistency was found between groups; inter-rater agreement was not significantly different on the ADI-R and only one significant difference for an item on the ADOS was found. Further, high parent satisfaction was reported for both conditions. Another study utilized telehealth assessment procedures to assess autism in young children (Corona et al., 2021). After randomized group assignment, remote assessors provided 


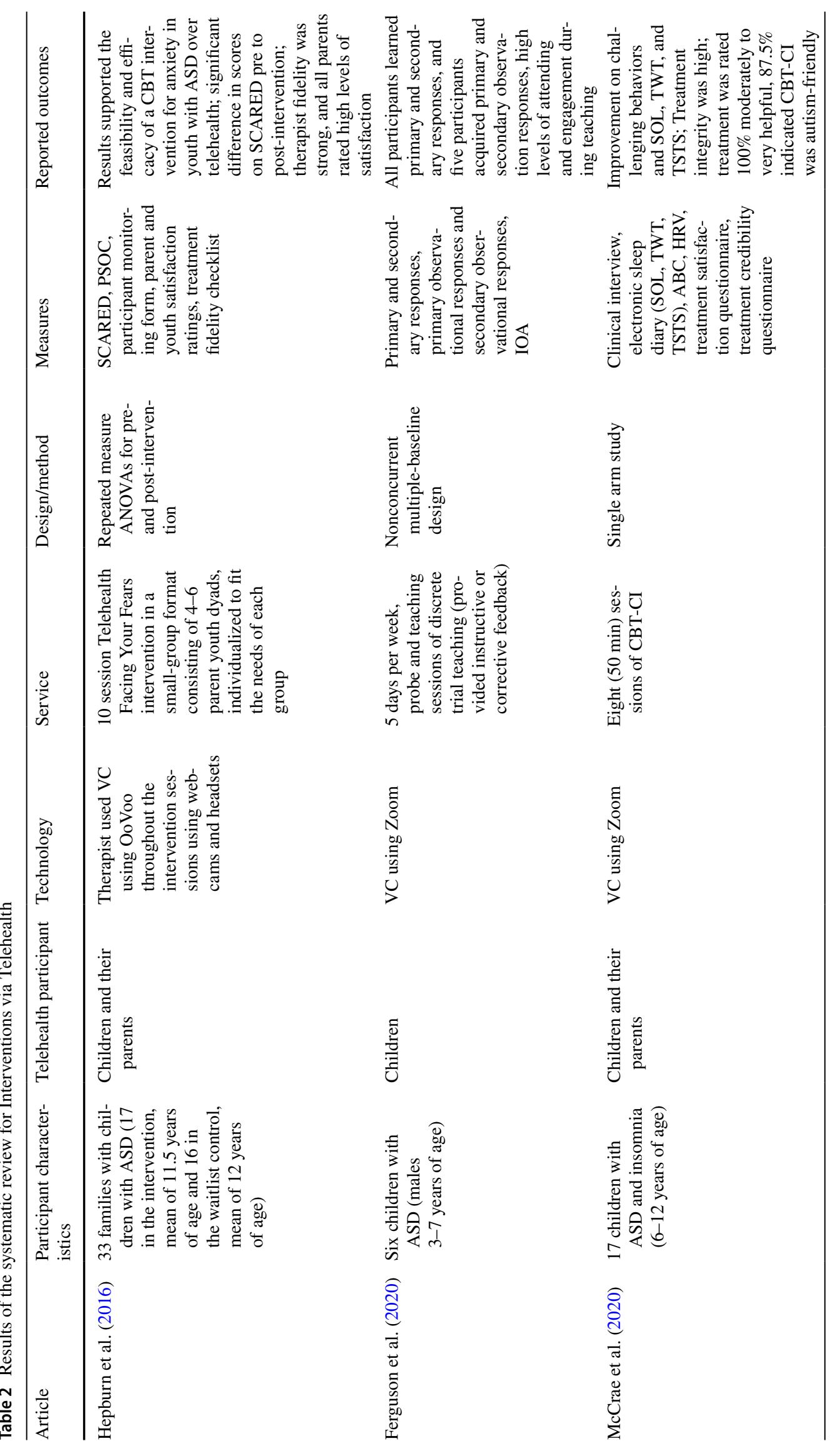




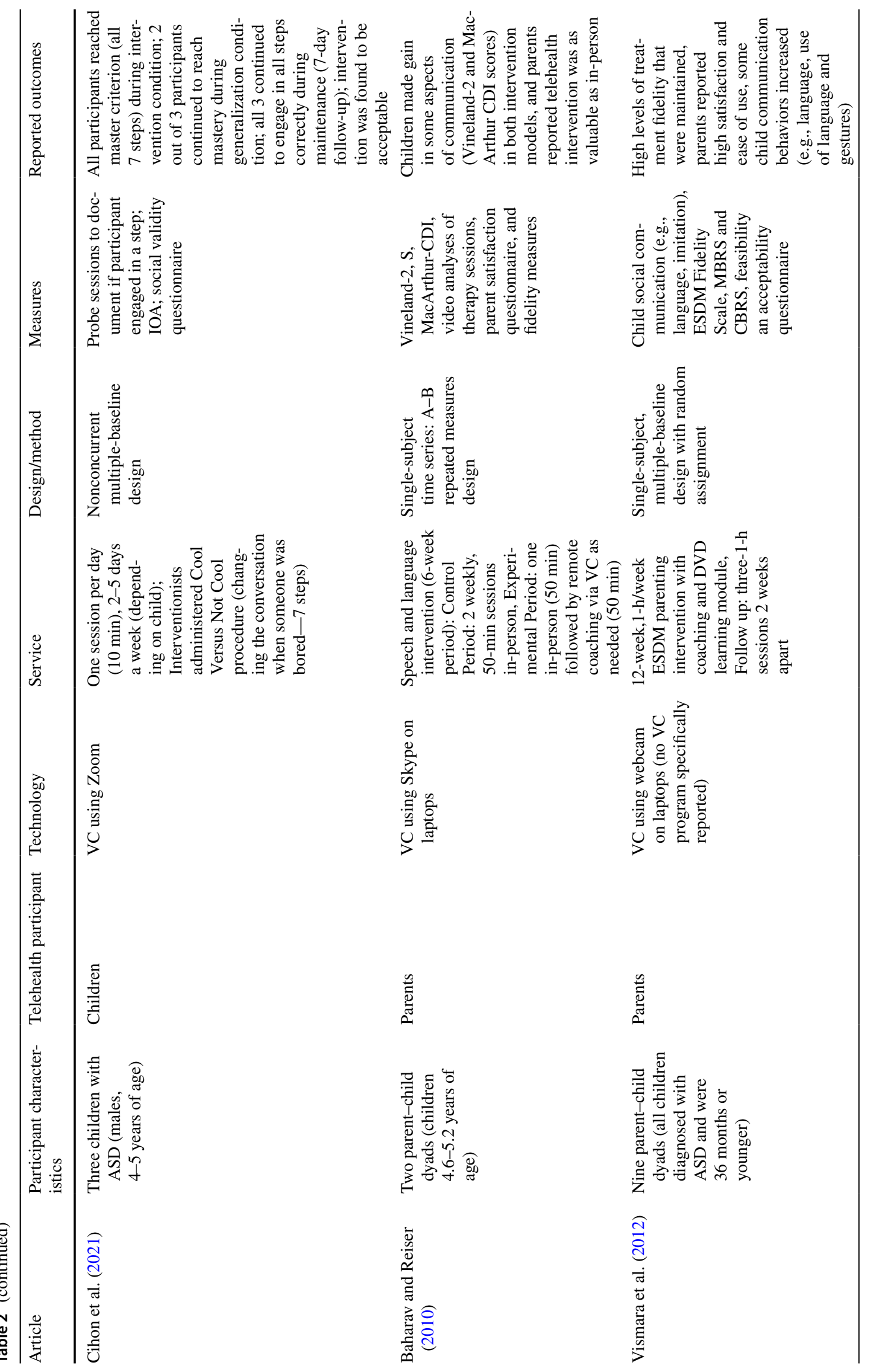




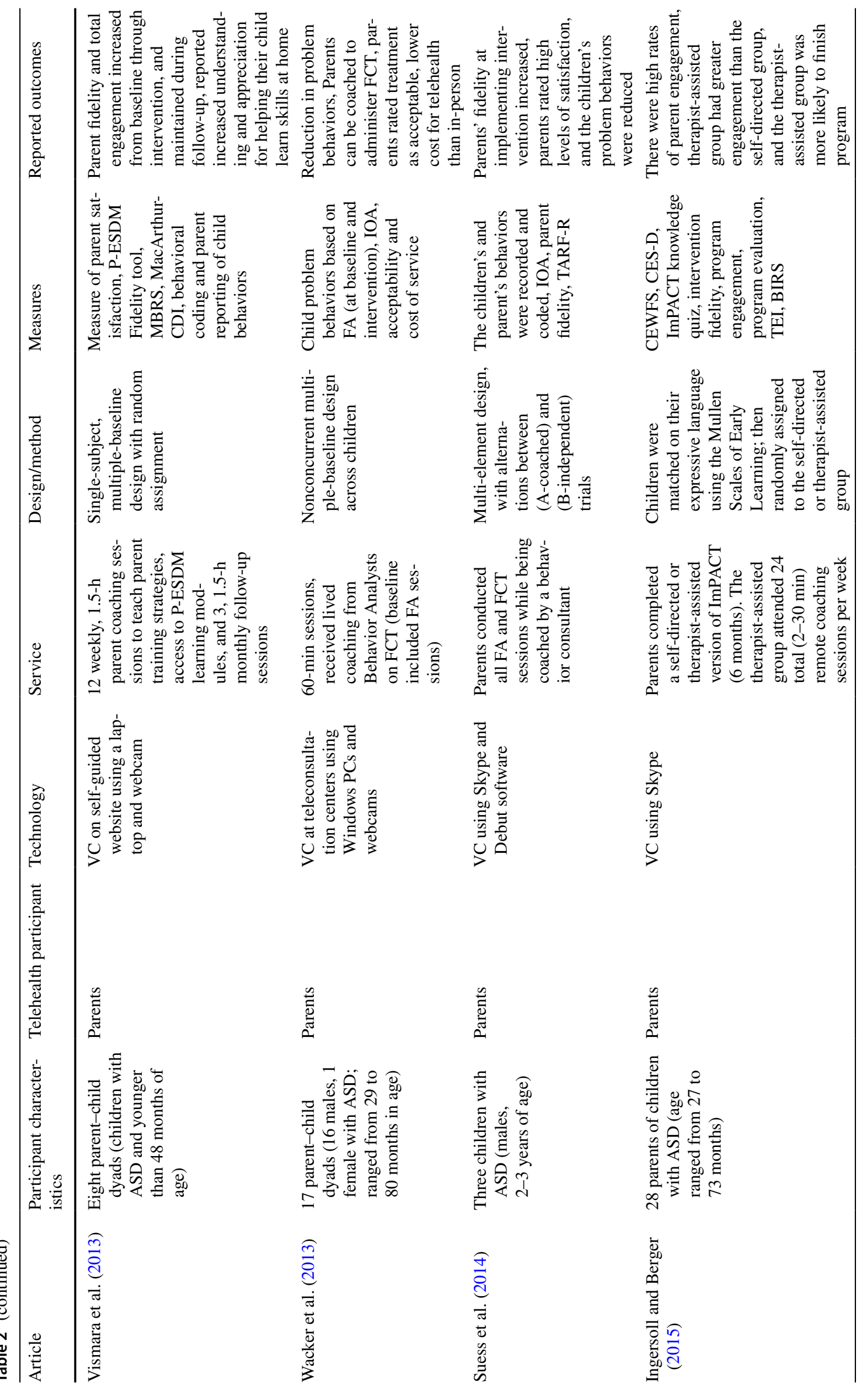




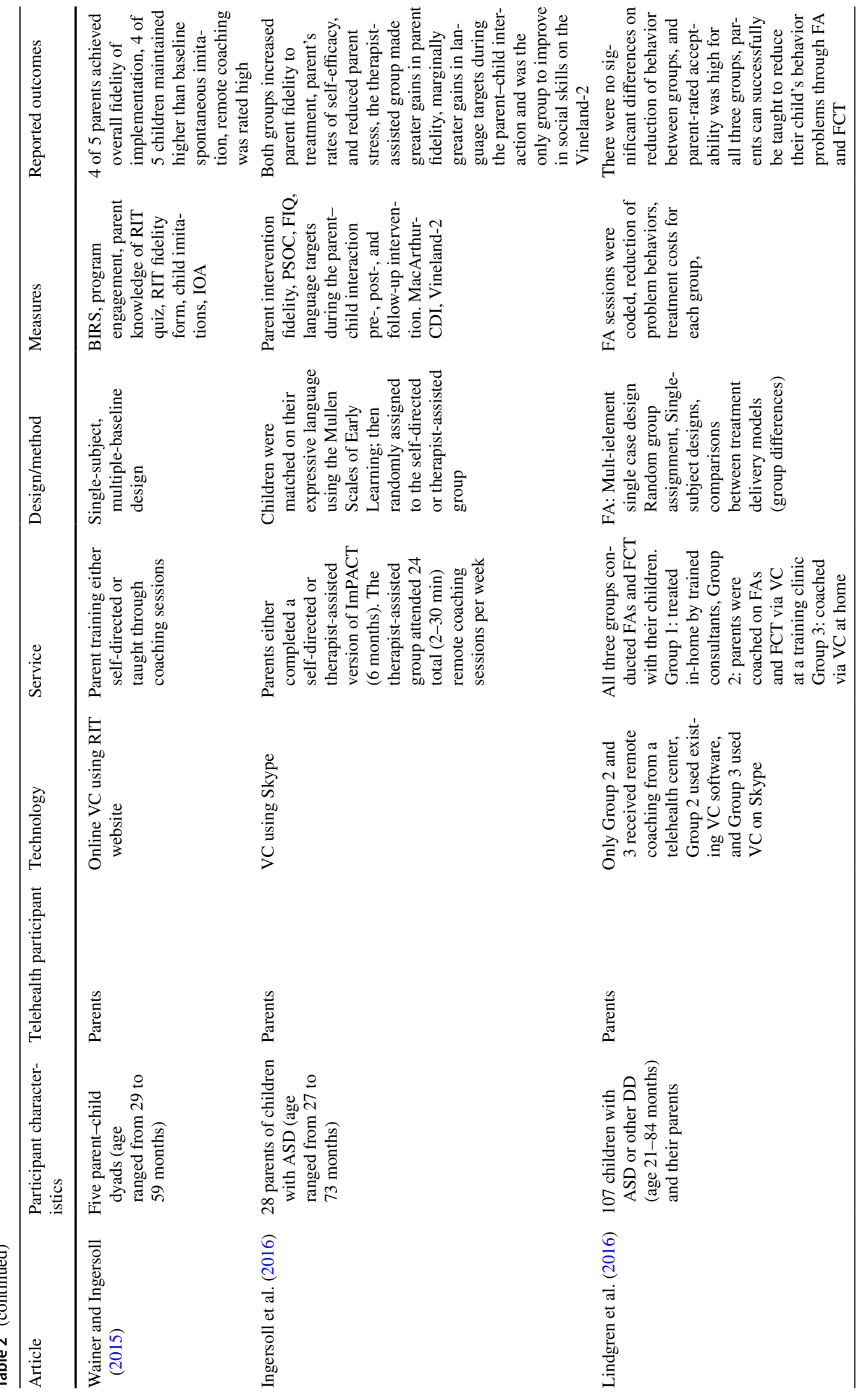




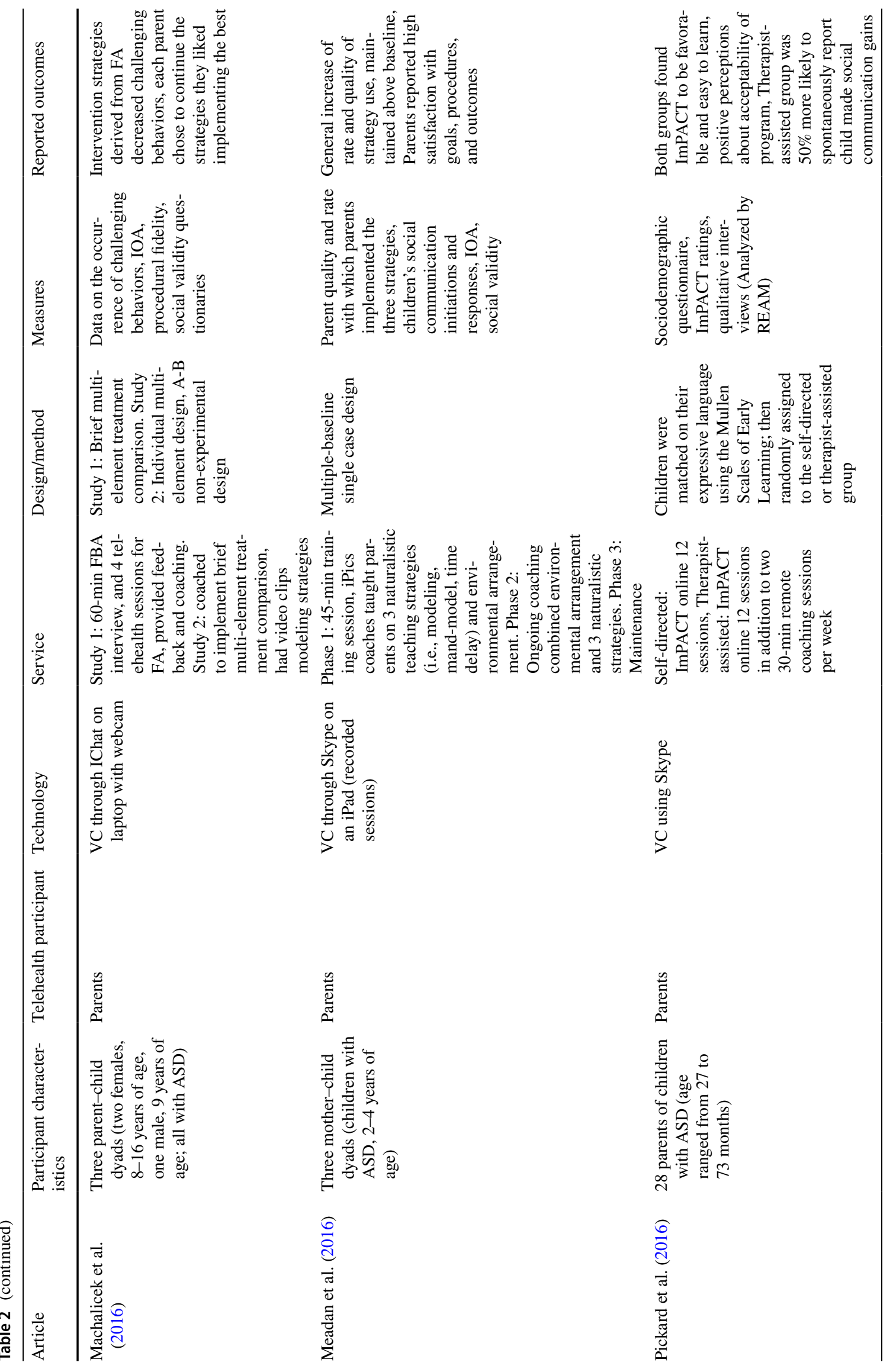




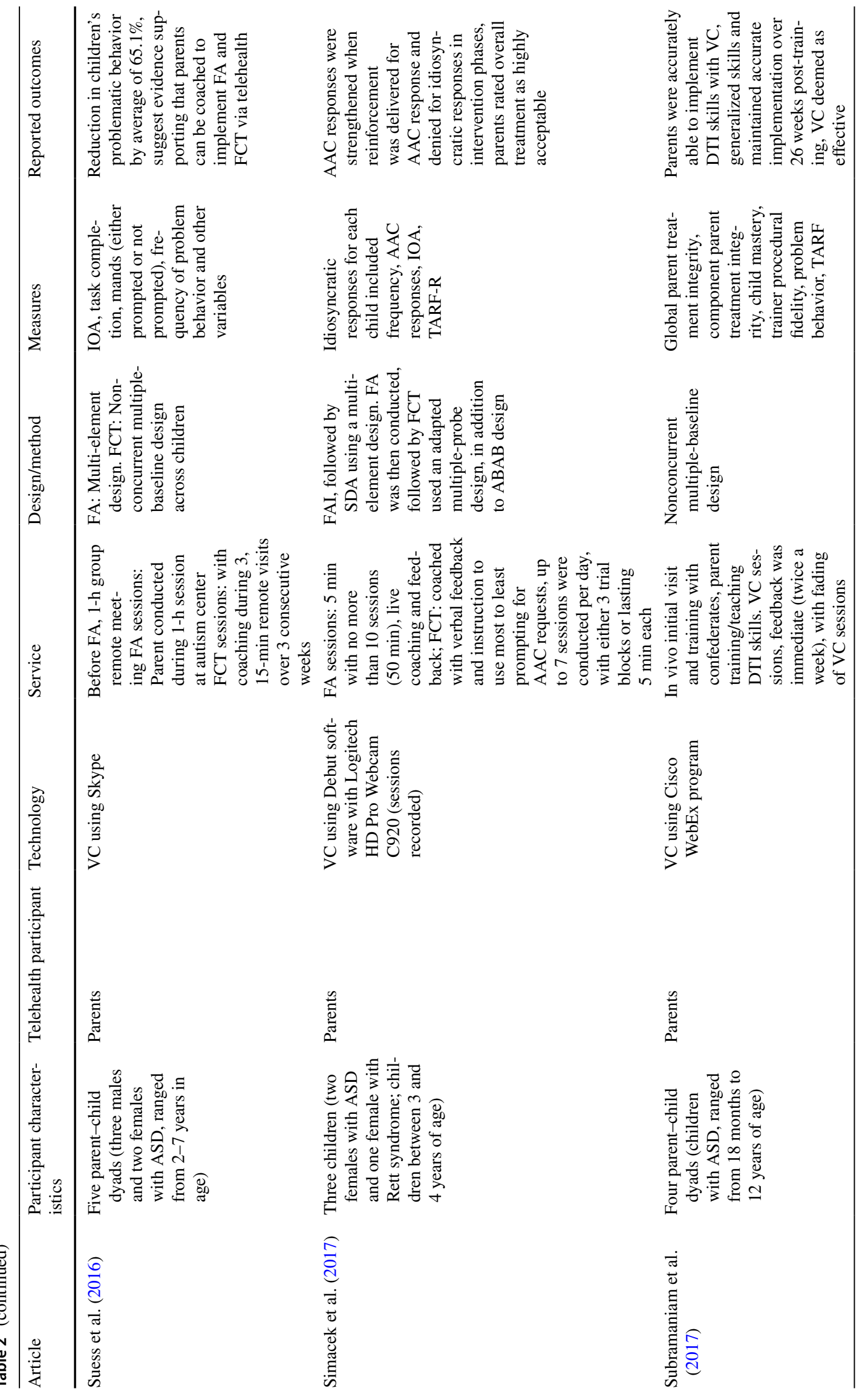




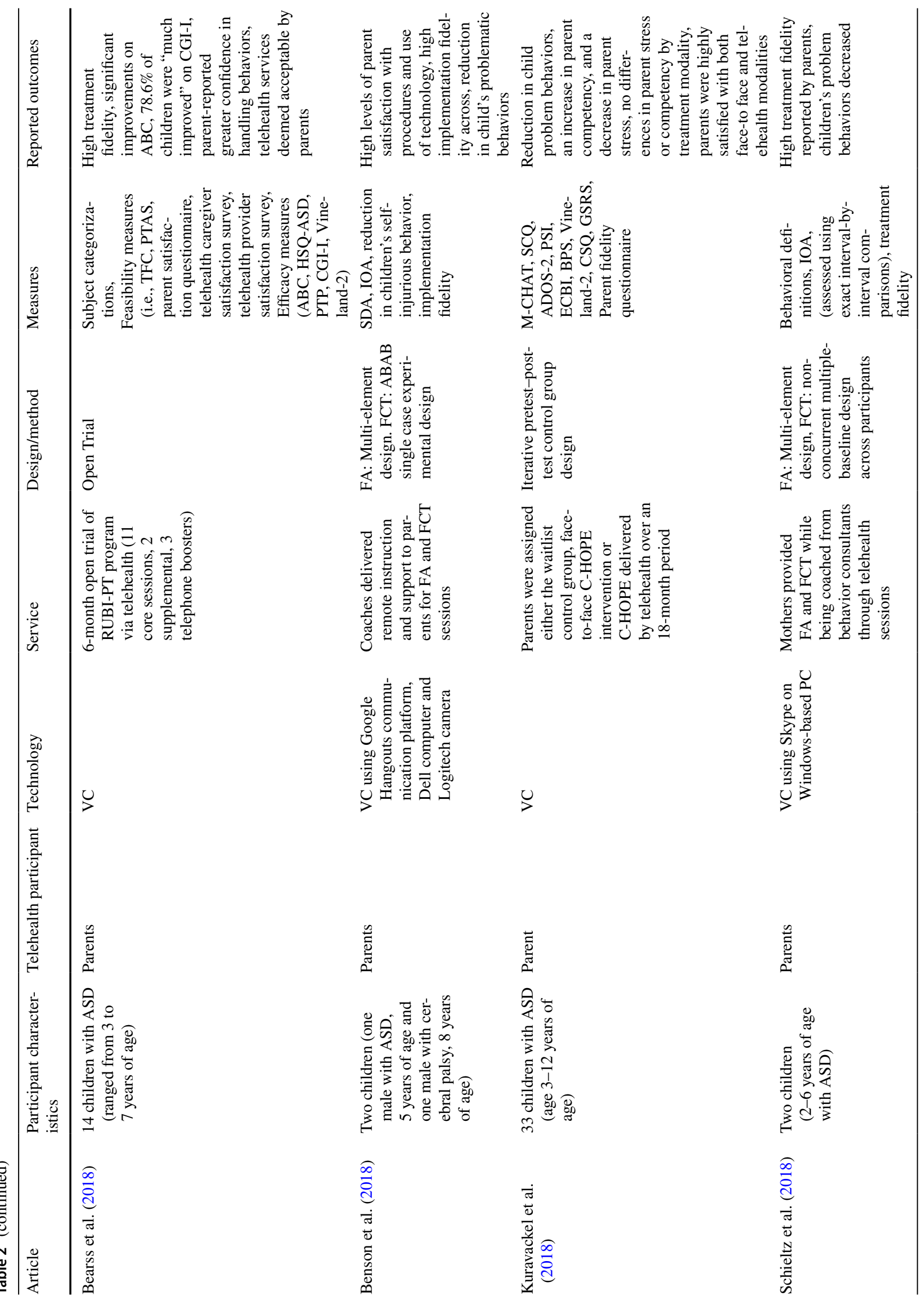




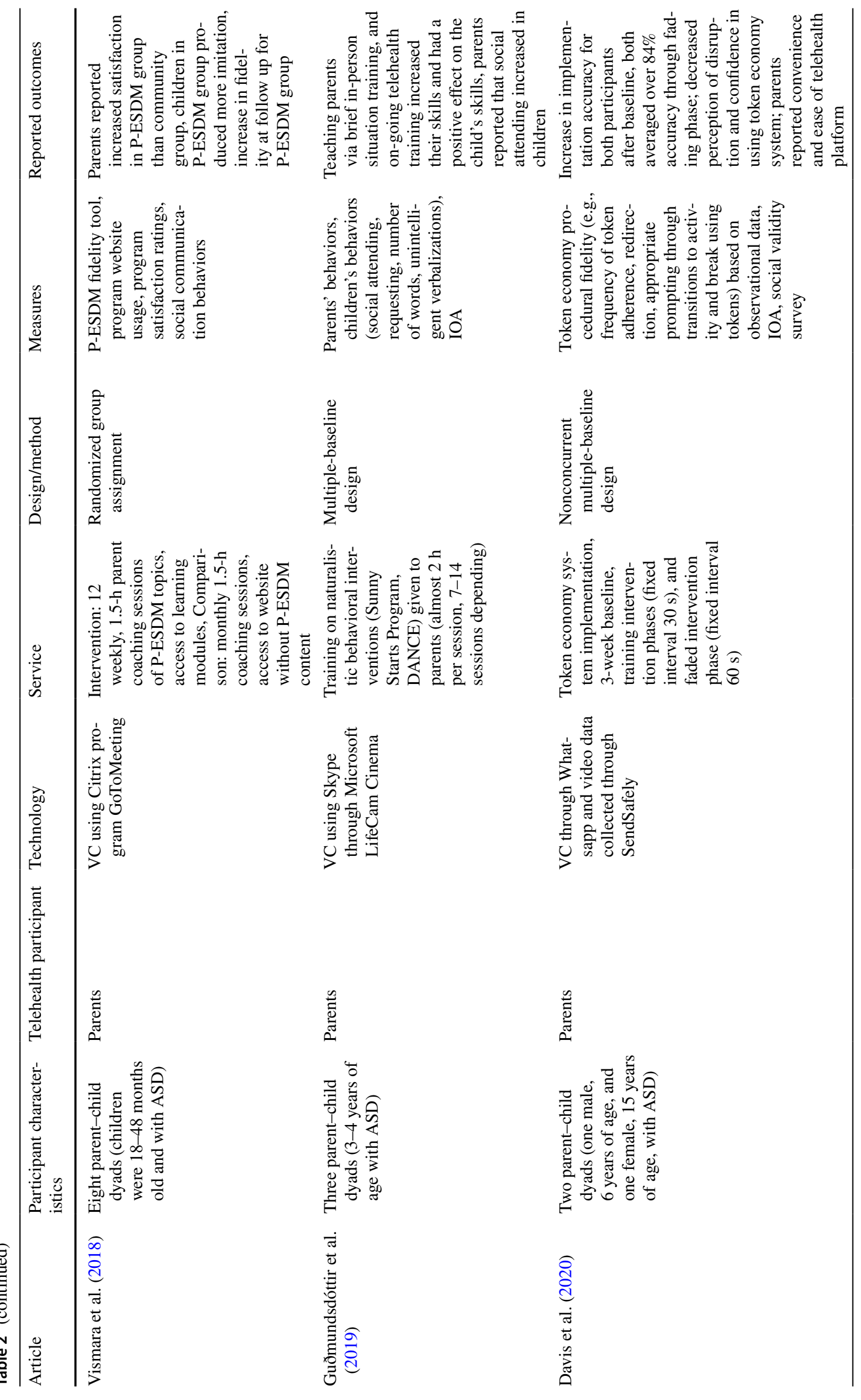




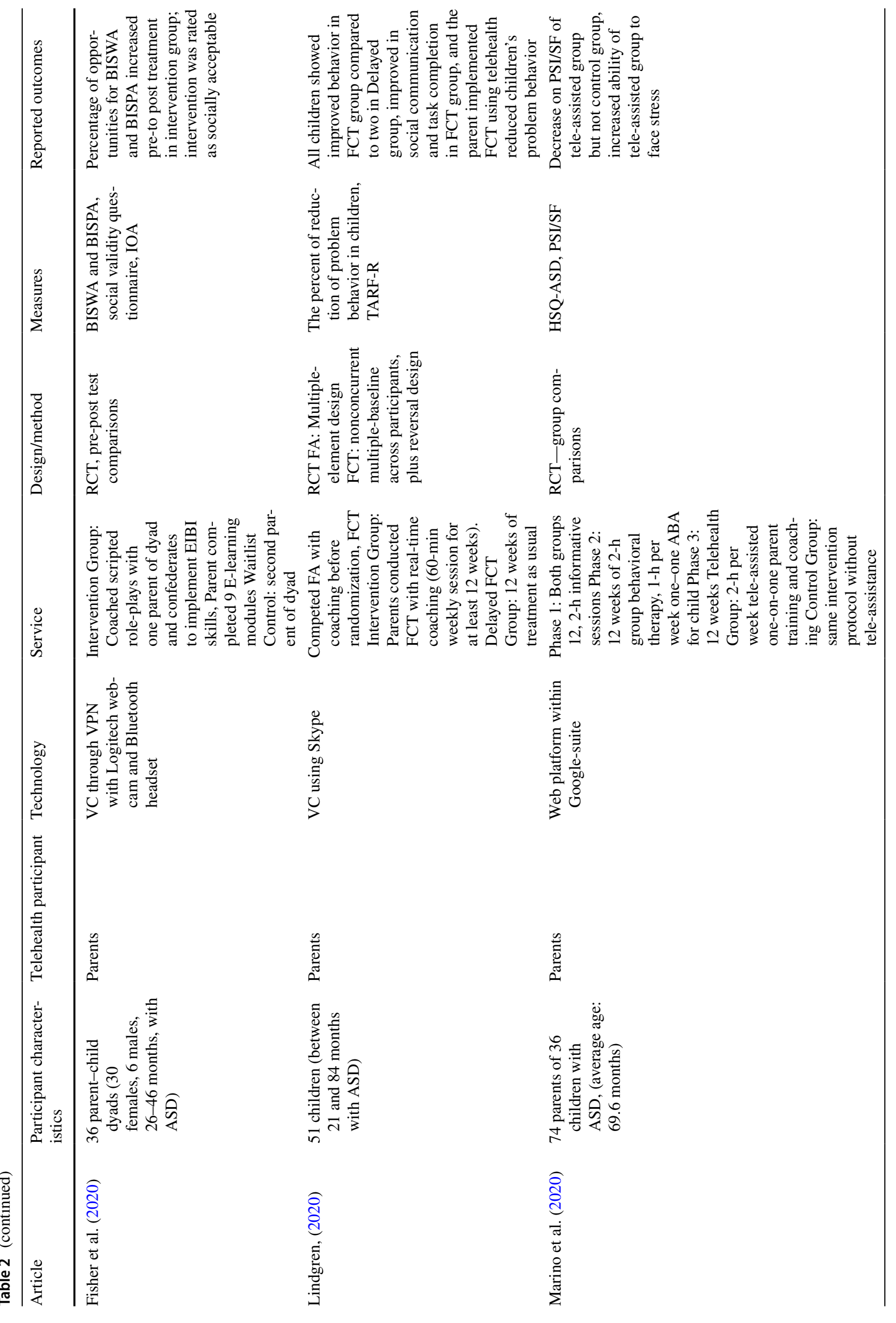




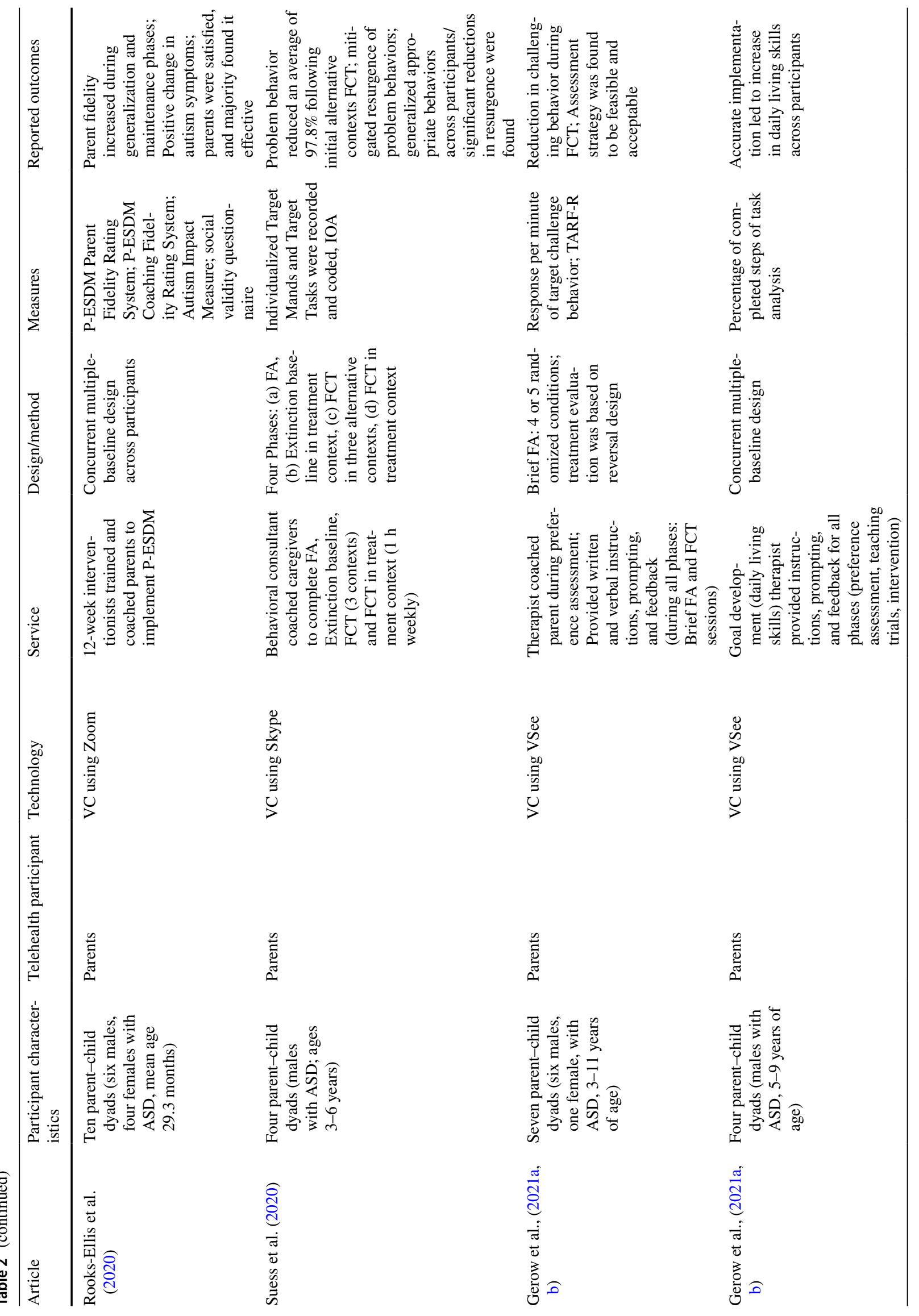




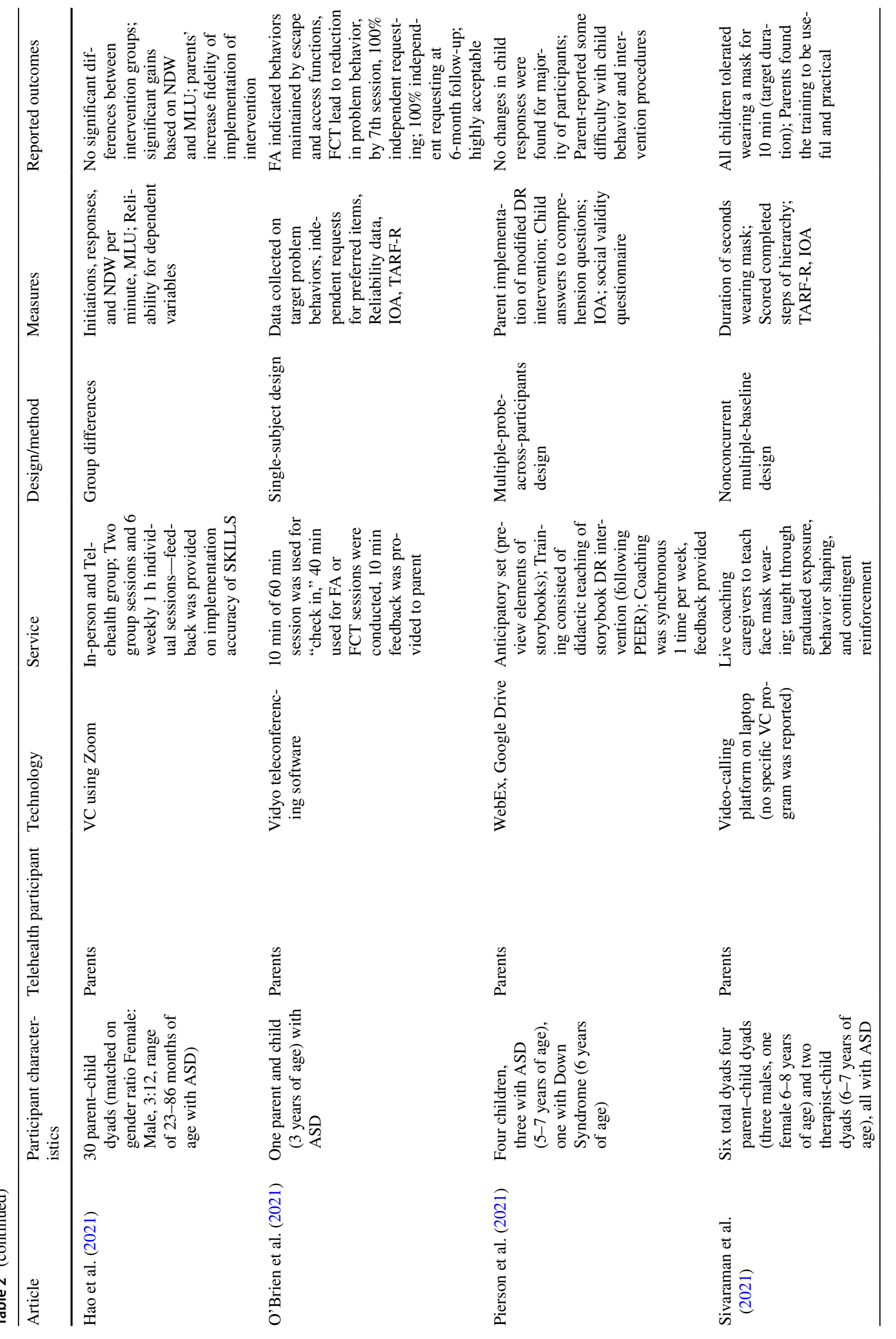




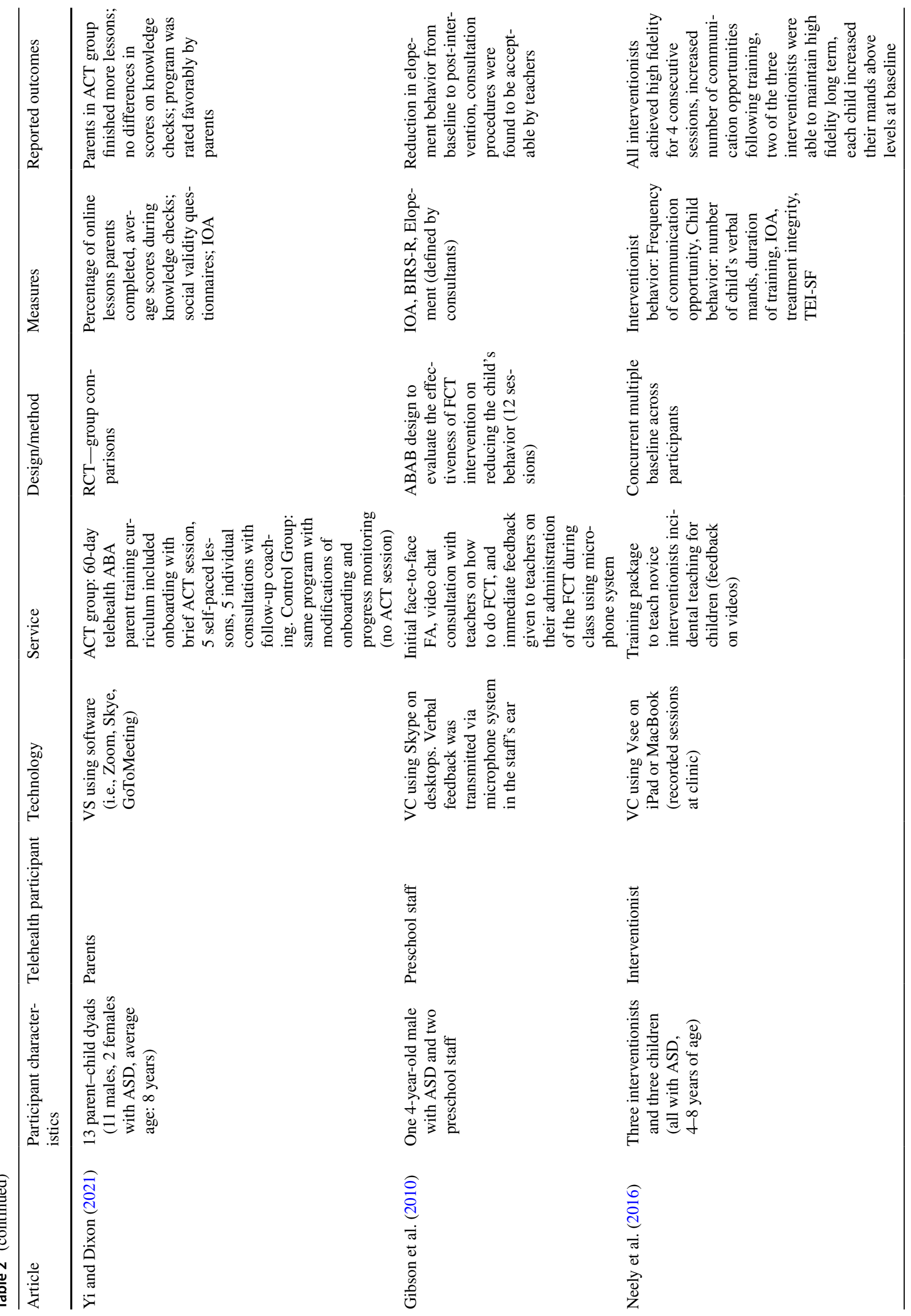




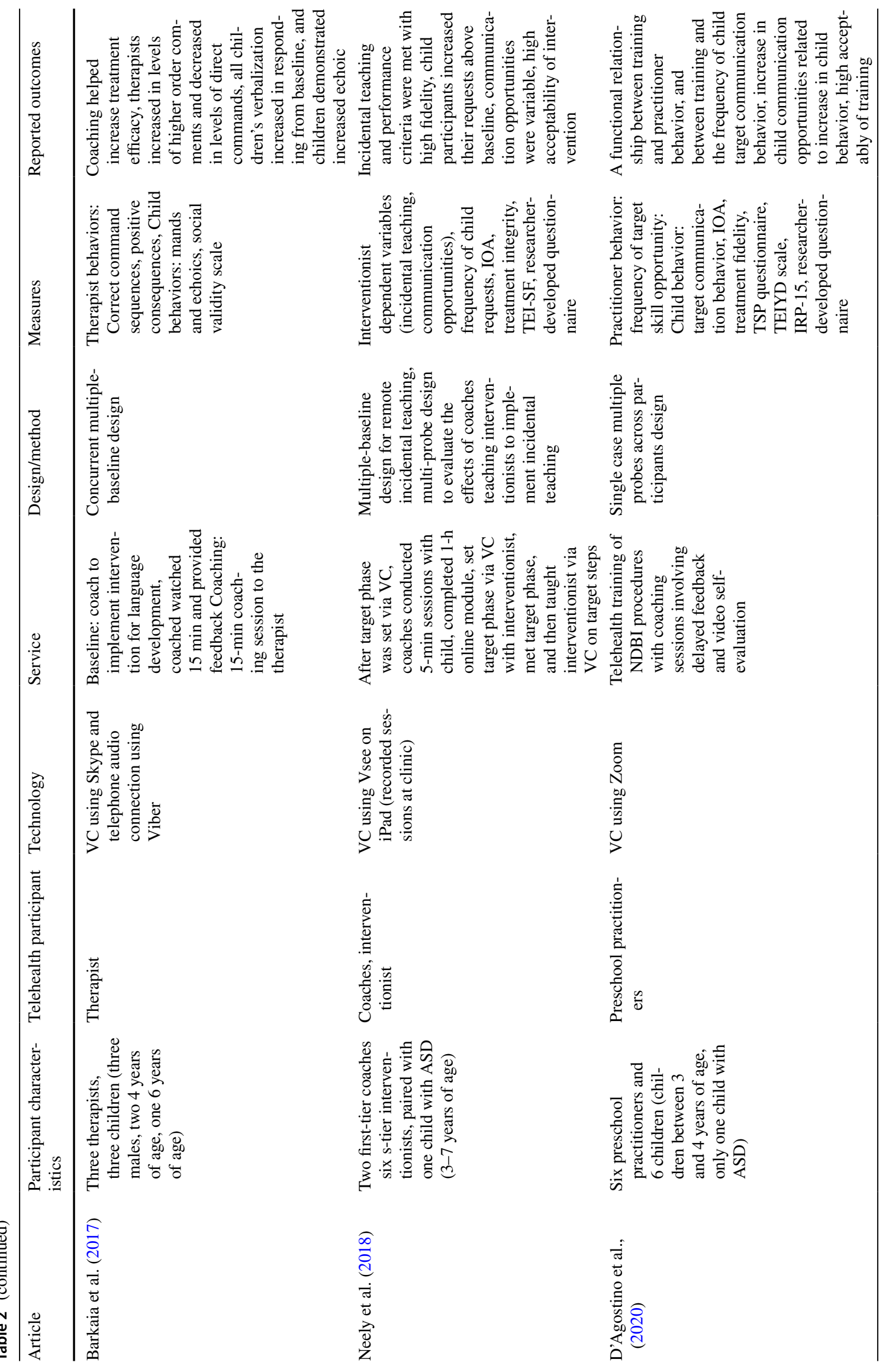




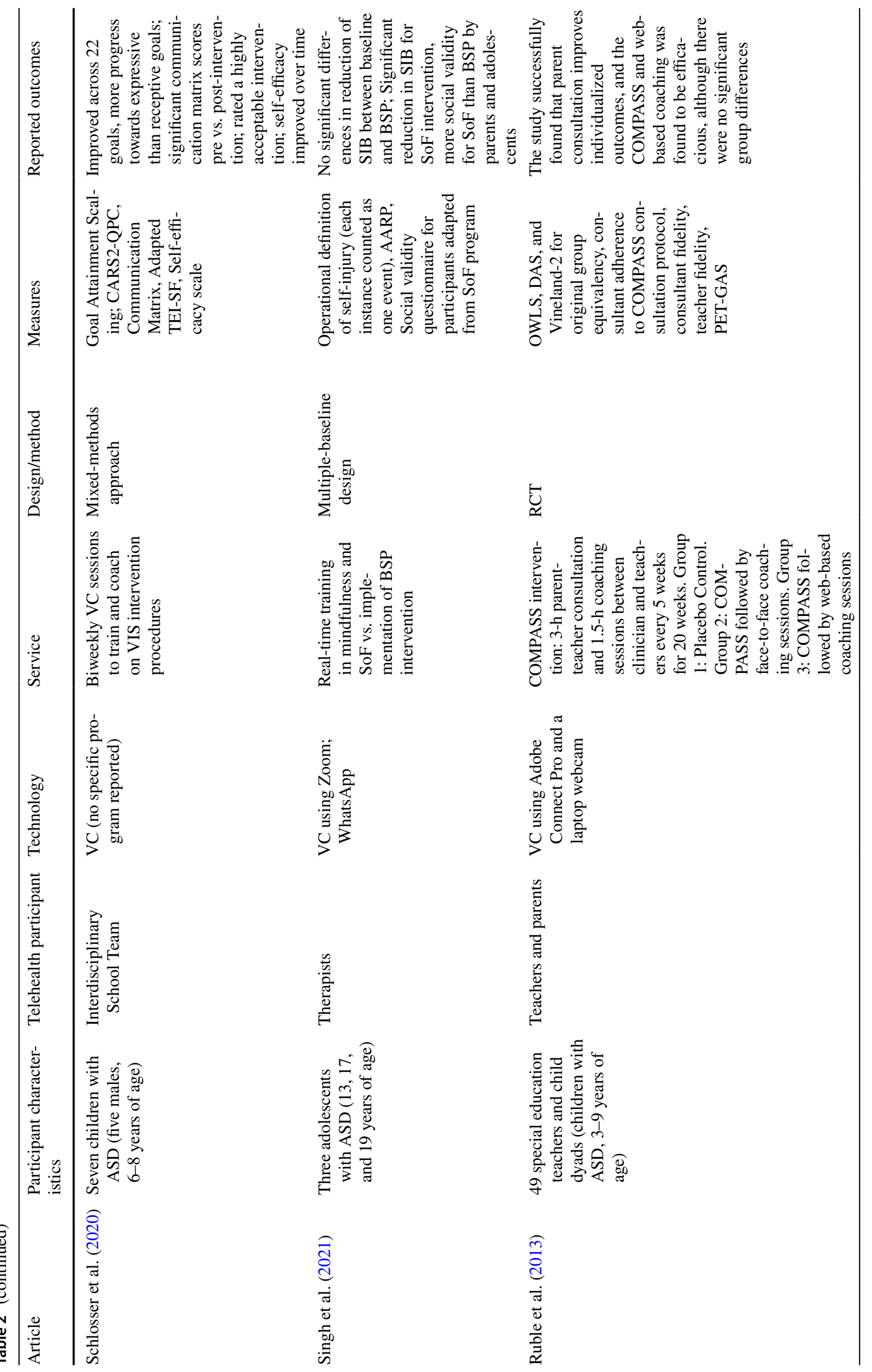




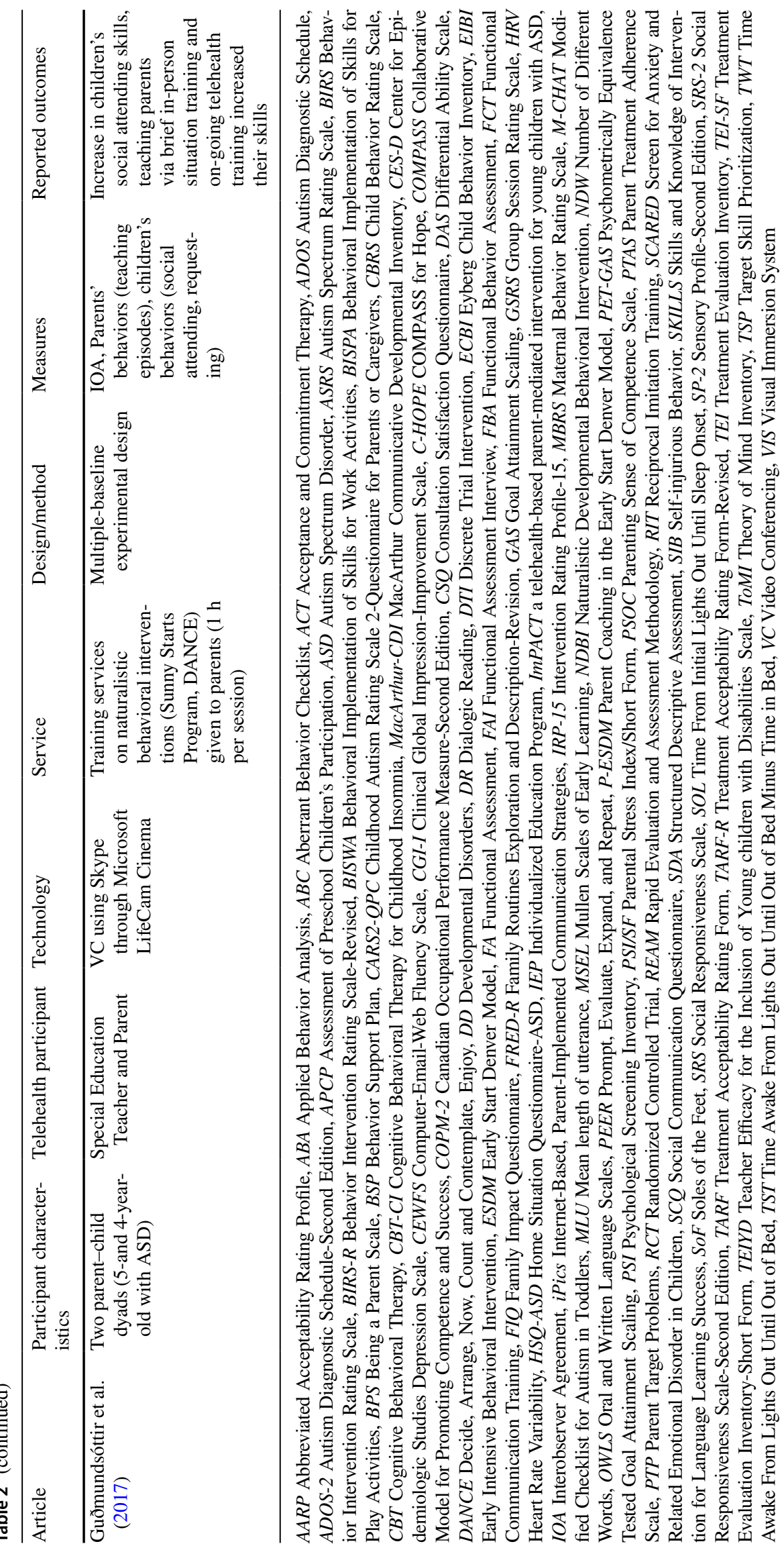


prompts to parents using an adaptation of the Screening Tool for Autism in Toddlers and Young Children (Stone et al., 2000) via VC or used the TELE-ASD-PEDS to guide parents to lead specific social tasks with their children (Corona et al., 2021). Both of these telehealth administrations were used to establish diagnostic accuracy; across the sample, diagnostic accuracy was $86-77 \%$ of parents reported that they would prefer both to play and observe the child during the remote assessment instead of just playing with the child or just observing (Corona et al., 2021). Based on these two studies, it does appear that well-established diagnostic measures of ASD (i.e., ADOS and ADI-R), as well as other measures of ASD symptomology, can be used via telehealth successfully as accurate alternatives for identifying children with ASD rather than solely relying on in-person assessments (Corona et al., 2021; Reese et al., 2013).

Lastly, one study used a telehealth platform to educate, train, and coach a parent at a teleconsultation center to implement a functional analysis assessment (four conditions) with their children with ASD successfully (Wacker et al., 2013). Remote real-time coaching was effective in having parents administer the functional analysis and was successful with identifying the social function of the behaviors consistently (Wacker et al., 2013). Results were comparable to previous functional analysis studies where theses assessments were conducted in-home with parents but the telehealth administration was a more cost-effective strategy (Wacker et al., 2013).

\section{Telehealth Participant: Interventionists/Staff/Teachers of Children/Adolescents with ASD}

Four studies focused on implementing an assessment via telehealth with interventionists, staff, and/or teachers who work with individuals with ASD. Three studies utilized either teachers, direct-care staff, or clinical staff for the purposes of either implementing preference assessments with children with ASD (Ausenhus \& Higgins, 2019; Higgins et al., 2017; Machalicek et al., 2009), while one study used teachers to conduct functional analyses for challenging behavior in children with ASD (Machalicek et al., 2010).

A preference assessment is a structured method utilized to identify highly preferred items that can be used as reinforcers when teaching new skills (Ausenhus \& Higgins, 2019). Two studies utilized trained confederates to assist in the training of the staff to deliver the preference assessment to minimize the actual participant with ASD's exposure to an assessment of low treatment integrity (Ausenhus \& Higgins, 2019; Higgins et al., 2017). One study utilized delayed video feedback and real time feedback during roleplays with the confederate (Higgins et al., 2017), while the other provided remote, real-time feedback during the preference assessment with the participant with ASD (Ausenhus \& Higgins, 2019).
These studies found that training of staff to deliver preference assessments can be effectively done via telehealth and all trained staff indicated they would recommend this training procedure to others in the future. Further, procedural integrity was high across studies. The third study utilized $\mathrm{VC}$ to supervise teachers conducting paired-choice preference assessments with immediate remote feedback following each trial during the assessments (Machalicek et al., 2009). The teachers completed these preference assessments with $100 \%$ accuracy following feedback; when preference assessment results (the items that were selected as preferred) were further tested with the same children with ASD, the children did prefer the items selected during the preference assessment (Machalicek et al., 2009). Teachers and staff indicated satisfaction with the telehealth supervision protocol (Ausenhus \& Higgins, 2019; Higgins et al., 2017; Machalicek et al., 2009). Lastly, functional analyses were conducted with teachers of children with ASD using immediate feedback via $\mathrm{VC}$ and the supervision that was provided aided teachers in continuing to implement FAs effectively nine weeks after the initial feedback, but during the maintenance phase, the implementation for each teacher varied (Machalicek et al., 2010). This indicates that remote functional analyses conducted by teachers may be an acceptable tool, but more follow-up sessions may be needed after the initial sequences of training to ensure accurate implementation.

Based upon this review, there are a limited number of studies that examine the efficacy of the assessment of ASD or the use of other types of assessments with children and adolescents with ASD via telehealth. Of the articles that examined social validity, the use of technology and equipment was acceptable and the technical difficulties experienced, if any, were reportedly manageable (Ausenhus \& Higgins, 2019; Higgins et al., 2017; Machalicek et al., 2009, 2010). Primarily, all of the assessment studies included in this review demonstrate the feasibility of using telehealth to accurately assess not only for diagnostic purposes, but to also conduct other forms of assessments with children with ASD.

\section{Treatment}

Forty-seven studies in this review provided a variety of intervention services via telehealth to individuals with ASD. Of the 47 studies, four studies targeted the child with ASD directly, 34 had the parent receiving the telehealth intervention and then the parent administered it with their child with ASD, seven utilized other individuals (i.e., teachers, therapists, interventionists), and two studies used both a parent and teacher. Similar to the assessment section, VC was the telehealth method utilized the most across studies. 


\section{Telehealth Participant: Children and Adolescents with ASD}

Four studies focused on implementing an intervention via telehealth directly with individuals with ASD. The VC platform was either OoVoo (Hepburn et al., 2016) or Zoom Video conferencing (Cihon et al., 2021; Ferguson et al., 2020; McCrae et al., 2020). Two studies utilized telehealth to administer cognitive behavioral therapy directing to children with ASD (Hepburn et al., 2016; McCrae et al., 2020). The first study delivered a cognitive behavioral intervention (modified version of Face Your Fears: Group Therapy for Managing Anxiety in Children with High Functioning Autism) via VC for 10 sessions to small groups of childparent dyads to address co-occurring anxiety symptoms in ASD (Hepburn et al., 2016; Reaven et al., 2011). The telehealth group had significantly lower parent-reported youth anxiety symptoms than the waitlist control group following the completion of the study. Parents and youth indicated strong satisfaction with the telehealth delivery method, although technical glitches (e.g., lost connections) were commonly reported (Hepburn et al., 2016). The second study conducted cognitive behavior therapy for childhood insomnia for eight, 50 min sessions with children with ASD and insomnia (McCrae et al., 2020). Children with ASD saw improvements in challenging behaviors and sleep related measures (e.g., time awake from lights out until out of bed) and reported the intervention to be moderately to very helpful (McCrae et al., 2020). Treatment integrity for both studies was high (Hepburn et al., 2016; McCrae et al., 2020).

An additional study employed discrete trial teaching with dyads of children with ASD, five days per week, with both real-time instructive feedback or corrective feedback (Ferguson et al., 2020). High levels of attending and engagement were found during teaching and all participants acquired both primary responses (defined as when participant vocally stated the name of the stimulus when the stimulus was presented) and secondary responses (defined as when the participant vocally responded to a question about the stimulus when the stimulus was presented) (Ferguson et al., 2020). Lastly, a social skills intervention (the Cool Versus Not Cool intervention) was implemented via telehealth with three children with ASD; this intervention targeted the social skill of changing the conversation by recognizing when someone was bored (Cihon et al., 2021). The social skills intervention had seven steps to achieve mastery and all three participants mastered all of the steps by the end of the intervention program, whereas only two continued to reach mastery during generalization phase (Cihon et al., 2021). All four of these studies are indicative of progress in implementing interventions directly to individuals with ASD via telehealth platforms.
Telehealth Participant: Parents of Children and Adolescents with ASD

Thirty-four studies in this review featured the parents of individuals with ASD as the primary receiver of the telehealth intervention programs, which then they delivered to their children with ASD. All of the studies used VC as the main form of communication to deliver the interventions, but the software used varied (e.g., Skype, Zoom, Cisco WebEx, GoToMeeting, Google Hangouts, Whatsapp, Vsee, and Vidyo). For clarity and discussion purposes, the 34 studies are divided by intervention type below.

Behavior Management/Parenting Training Eleven studies featured telehealth delivered parent management/parent training interventions and all utilized VC through different telehealth platforms. Three studies evaluated the effectiveness of ImPACT, a telehealth-based parent-mediated intervention for children with ASD, implemented via selfdirected and therapist-assisted delivery models (Ingersoll \& Berger, 2015; Ingersoll et al., 2016; Pickard et al., 2016). These studies all found that parents were engaged in both conditions but therapist assistance increased engagement and acceptability of the program; initial findings also support an increase in social communication skills (Pickard et al., 2016) and improved language targets (Ingersoll et al., 2016) regardless of condition, but only the therapistassisted group increased in social skills (Ingersoll et al., 2016). Similarly, four studies explored the feasibility and acceptability of parent implemented interventions based on the Early Start Denver Model (ESDM) and the effect on different child behaviors (e.g., communication, imitation) (Rooks-Ellis et al., 2020; Vismara et al., 2012, 2013, 2018). Across all four studies, parent fidelity in implementation increased through intervention and maintained through follow-up. Autism symptoms, as measured by the Autism Spectrum Rating Scale, decreased (Rooks-Ellis et al., 2020) and child language and use of both language gestures increased (Vismara et al., 2012). Overall, the use of the ESDM was an effective intervention delivered via telehealth. Additionally, the use of a telehealth hybrid of reciprocal imitation training (RIT) similarly found high parent acceptability and effectiveness in increasing their child's spontaneous imitation skills (Wainer \& Ingersoll, 2015). Two other specific parent-mediated intervention programs were investigated. The Research Unit on Behavior Interventions-Parent Training (RUBI-PT) program was administered via telehealth over the course of 16 weeks, followed by three booster sessions, to address a variety of problem behaviors (Bearss et al., 2018). All caregivers indicated more confidence in handling problem behaviors and all families who completed the telehealth satisfaction questionnaire indicated that they would 
recommended the telehealth program to other parents (Bearss et al., 2018). The Sunny Starts Program, a parent training program, used naturalistic teaching strategies to increase specific child behaviors (i.e., social attending and requesting skills) (Guðmundsdóttir, 2019). After an initial in-person training, all communication and sessions were conducted via telehealth (Guðmundsdóttir et al., 2019). Social attending increased and skills were maintained at last 1 month after follow-up and generalization varied across the three parent-child dyads (Guðmundsdóttir et al., 2019). Lastly, Davis et al. (2020) utilized telehealth to teach parents to implement a token economy system (30-second fixed intervals) with children with ASD. Parents were able to implement the reinforcement system with over $84 \%$ accuracy through the faded intervention phase and parents reported that the telehealth platform was convenient and easy to use (Davis et al., 2020).

Functional Communication Training An additional twelve studies utilized telehealth via VC to deliver functional communication training (FCT) after functional analyses that were also delivered via telehealth (Benson et al., 2018; Gerow et al., 2021a, b; Lindgren, 2016, 2020; Machalicek et al., 2016; O'Brien et al., 2021; Schieltz et al., 2018; Simacek et al., 2017; Suess et al., 2014, 2016, 2020; Wacker et al., 2013). Studies varied on the design and method used to evaluate the implementation of the FCT (e.g., randomized control trial, single-subject design) but the coaching of parents via VC during functional analysis and FCT sessions was utilized across studies. After the implementation of FCT with remote coaching and training via telehealth, child problem behaviors were reduced in 11 studies (Benson et al., 2018; Gerow et al., 2021a, b; Lindgren et al., 2016, 2020; Machalicek et al., 2016; O'Brien et al., 2021; Schieltz et al., 2018; Suess et al., 2014, 2016, 2020; Wacker et al., 2013). One particular study focused on reducing the resurgence of problem behaviors once they were reduced significantly and demonstrated that remote coaching of FCT can accomplish this both effectively and more rapidly than previous research (Suess et al., 2020). Further, parents were able to conduct a functional analysis and the intervention strategies derived from this reduced the child's problem behaviors (Machalicek et al., 2016; Suess et al., 2016). The use of FCT with coaching via telehealth was also used to increase the number of augmentative and alternative communication requests in children with ASD; the use of differential reinforcement enhanced the augmentative and alternative communication responses (Simacek et al., 2017). High level of satisfaction with telehealth implementation and interventions were found to be acceptable across studies (Benson et al., 2018; Gerow et al., 2021a, b; Schieltz et al., 2018; Suess et al., 2014; Wacker et al., 2013).Overall, it appears that parents are able to learn and implement both functional analysis and FCT with children with ASD successfully via telehealth.

Social Communication Interventions Three studies specifically used parents to implement social communication intervention programs in children with ASD (Baharav \& Reiser, 2010; Hao et al., 2021; Meadan et al., 2016). The study by Meadan et al., 2016, adapted the Parent-Implemented Communication Strategies (PiCS) program, which is an in-person treatment, to become an internet-based program (i-PiCS) that was taught remotely with coaching sessions conducted via a telehealth platform. They investigated whether a parent's application of the naturalistic teaching strategies that were taught and coached via $\mathrm{VC}$ would increase their child with autism's expressive communication skills. Parents learned to successfully implement the strategies only when they were coached via $\mathrm{VC}$ and saw increases in child's social communication skills (Meadan et al., 2016). Similarly, another study examined group differences between an in-person and telehealth implementation of the Skills and Knowledge of Intervention for Language Learning Success (SKILLS) project with parents and children with ASD (Hao et al., 2021). There were six, 1-h sessions across 6 weeks, where parents would be coached and provided feedback on skills implementation with their children. Hao et al. (2021) found increases in child's number of different words and mean length utterance but did not find significant group differences between outcomes, indicating that the telehealth implementation can be effective. Lastly, the traditional in-person model (2 weekly sessions) was compared to a hybrid model (one in-person followed by one remote session via telehealth with coaching) in the effectiveness of delivering a speech and language intervention; children made communication gains in both (Baharav \& Reiser, 2010). Parents also reported high satisfaction with the intervention (Baharav \& Reiser, 2010). All three studies concluded that parents were satisfied with the social communication intervention they received via telehealth and all three studies demonstrated gains in social communication skills when the intervention was delivered via telehealth; therefore, there is support for the use of telehealth in the implementation of social communication interventions with children with ASD.

Applied Behavior Analysis (ABA) Four studies examined the possibility of using ABA across telehealth platforms (Fisher et al., 2020; Marino et al., 2020; Subramaniam et al., 2017; Yi \& Dixon, 2021) One study utilized VC to aid parents via telehealth to deliver discrete-trial instruction (DTI), an intervention based upon applied behavior analysis (ABA), with their children with ASD (Subramaniam et al., 2017). Parents first received necessary in-person behavioral skills training on how to implement the DTI procedures and then trainers 
conducted VC sessions with the parents and provided immediate feedback after each session; parents continued to have high treatment integrity with remote supervision months following the initial training (Subramaniam et al., 2017). While this study demonstrated that DTI can be implemented with feedback to parents via telehealth, there are limitations that need to be further explored (e.g., adherence to scheduled meetings, use of in session coaching in addition to end of session feedback) to determine if DTI can successfully implement over time via telehealth. Marino and colleagues (2020) examined the effect of an in-person versus telehealth assisted behavioral intervention for children with ASD. Participants were randomized for group assignment and the study was conducted through three phases. During Phase 1, all groups received twelve, 2-h informative sessions on ABA and characteristics of ASD. During Phase 2 all parents received 2-h weekly group behavioral therapy and all children received $1 \mathrm{~h}$ per week of individualized ABA therapy for 12 weeks. Lastly, during Phase 3, for 12 weeks, parents in the telehealth group received 2-h per week one-on-one behavioral training and coaching while the control group received 2-h per week in-person treatment (Marino et al., 2020) Results demonstrated a decrease in parent-reported stress related to child's challenging behaviors and improvements in their perception on how to manage these behaviors was seen in the telehealth group only (Marino et al., 2020). Another study examined the efficacy of parent implementation of early intensive behavioral intervention skills via telehealth using parent-child dyads, where one parent received the intervention and other parent was in the control group (Fisher et al., 2020). Similarly, to Marino et al., 2020, Fisher et al. (2020) found increases in correct implementation of skills for both work and play activities in the intervention group only. Lastly, Yi and Dixon (2021) investigated the differences between a 60-day ABA intervention with and without an Acceptance and Commitment Therapy (ACT) session during onboarding. After the onboarding meeting, parents completed five self-paced online lessons each followed by a VC consultation session with follow-up coaching based on the behavior management plan created for each family. Parents in the ACT group completed more lessons and progressed further in treatment compared to the control group but did not significantly differ on the knowledge they gained throughout the study (Yi \& Dixon, 2021). All four studies reported that the parents indicated positive aspects of the telehealth intervention; some parents reported the telehealth intervention to be reasonable (Subramaniam et al., 2017), while the other studies who directly assessed social validity indicated high satisfaction with the delivery of the intervention through telehealth (Fisher et al., 2020; Marino et al., 2020; Yi \& Dixon, 2021).
Other Intervention Programs Four other studies utilized telehealth to deliver other parent-mediated intervention programs (Gerow, et al., 2021a, b; Kuravackel et al., 2018; Pierson et al., 2021; Sivaraman et al., 2021). The Collaborative Model of Promoting Competence and Success (COMPASS) for Hope was delivered via VC; this program led to a reduction of problem behaviors for the children with ASD as well as a decrease in parent-reported stress and an increase in parent-reported competency (Kuravackel et al., 2018). Similarly, another study focusing on daily living skills examined parent coaching (prompting and feedback from therapist) for skill implementation based on individualized goals delivered via telehealth and found the intervention increased daily living skills in children with ASD; more specifically, increases in the amount of completed steps occurred for 10 out of the 12 individualized skills addressed by the intervention (across participants) (Gerow, et al., 2021a, b). However, one study conducted by Pierson et al. (2021) did not find results supporting the use of their intervention in increasing specific child outcomes. A modified dialogic reading intervention was implemented via telehealth with parent training and coaching components, requiring the parents to provide prompts after the child provided his or her response to comprehension questions (Pierson et al., 2021). Very limited changes were found in the behavior of the children receiving the intervention; therefore, the child outcomes were not indicative of the intervention being helpful. Parents reported the intervention to be socially valid and the complex nature of this multi-element intervention may account for the lack of increases in children's comprehension (Pierson et al., 2021). Lastly, the study conducted by Sivaraman et al. (2021), which may be one of the most applicable studies for the current time period, examined the efficacy of a telehealth implemented mask wearing training for children with ASD. Live coaching via VC was used to guide parents through graduated exposures with their child with ASD putting on and wearing a mask; other intervention components included behavior shaping techniques and contingent positive reinforcement (Sivaraman et al., 2021). By the end of the intervention, all children with ASD were able to wear a mask for 10-min (the target duration) and parents reported the intervention to be useful (Sivaraman et al., 2021). This study demonstrated that with guidance from a professional via telehealth, other treatment providers as well as parents can learn how to successfully teach a child with ASD to wear a mask.

\section{Telehealth Participant: Interventionists/Staff/Teachers of Children and Adolescents with ASD}

Seven studies implemented telehealth interventions with either interventionists, staff, and/or teachers who work with 
individuals with ASD using a variety of VC platforms such as Skype, Zoom, Whatsapp, and VSee (Barkaia et al., 2017; D'Agostino et al., 2020; Gibson et al., 2010; Neely et al., 2016, 2018; Schlosser et al., 2020; Singh et al., 2021).

Behavior Interventions Four studies utilized behavioral interventions (D’Agostino et al., 2020; Neely et al., 2016, 2018; Singh et al., 2021). An incidental teaching training package taught remotely via $\mathrm{VC}$ to coaches and then interventionists, led to increased communication skills in the children with ASD and high fidelity in implementation (Neely et al., 2016, 2018). Naturalistic Developmental Behavior Intervention procedures, which incorporated selfpaced online modules, intervention planning, and video selfevaluation, were taught to preschool practitioners and then sessions via $\mathrm{VC}$ which involved delayed performance feedback (D'Agostino et al., 2020); practitioners achieved intervention fidelity and children with ASD increased in communication behaviors which both generalized and maintained at follow-up. Lastly, Singh et al. (2021) implemented both a behavioral program (behavioral support plan) and mindfulness-based program (Soles of the Feet) via telehealth to reduce the number of self-injurious behaviors (SIB) three children with ASD were engaging in. Results yielded a reduction in SIB when the children received the Soles of the Feet intervention but not when they only received the behavior support plan (Singh et al., 2021). Acceptability was high for each intervention across all four studies (D'Agostino et al., 2020; Neely et al., 2016, 2018; Singh et al., 2021).

Functional Communication Training Teachers were also utilized to implement FCT via videoconferencing with a behavioral consultant (e.g., with modeling, roleplays, and feedback) to reduce the elopement of a child with ASD (Gibson et al., 2010). High fidelity in implementation as well as high satisfaction with the implementation procedures yields additional evidence that providers can be trained to implement FCT via telehealth (Gibson et al., 2010).

Social Communication Interventions Two studies focused on utilizing teachers to aid in increasing social communication skills for children with ASD through the use of telehealth (Barkaia et al., 2017; Schlosser et al., 2020). Schlosser et al. (2020) investigated whether a coaching-based intervention, the Visual Immersion System ${ }^{\mathrm{TM}}$ (VIS ${ }^{\mathrm{TM}}$ ), can be implemented successfully via telehealth. The school's interdisciplinary team received a workshop on VISTM followed by biweekly VC sessions as well as in-between session communications via email or phone. Based on their primary outcome variable (goal attainment scaling) all participants improved individually based on their specified goals (overall made more progress on expressive language than receptive language goals) (Schlosser et al., 2020). Additionally, coaching via $\mathrm{VC}$ and telephone audio (via Viber) with a therapist was used to increase social attending and communication skills for a child with ASD (Barkaia et al., 2017). Both studies yielded high treatment acceptability and children benefited from increases in targets skills, which further supports the use of telehealth as a means to deliver social communication based interventions with children with ASD (Barkaia et al., 2017; Schlosser et al., 2020).

\section{Telehealth Participant: Parent AND Teacher of Children with ASD}

Two studies utilized both parents and teachers, together, to implement interventions for children with ASD. Specifically, one study utilized both a parent and special education teacher to increase social communication behaviors (e.g., requesting, attending) in children with $\mathrm{ASD}$; after in-person baseline assessments and initial training of the Sunny Starts Program, described earlier, communication and intervention sessions were conducted via telehealth (Guðmundsdóttir et al., 2017). Target child behaviors increased during intervention and follow-up phases (Guðmundsdóttir et al., 2017). A second study used parent-teacher consultations through the use of the Collaborative Model of Promoting Competence and Success (COMPASS) to examine the effects of web-based coaching compared to in-person coaching and a control condition (Ruble et al., 2013). There were no differences of overall change across goal domains for either the web-based or in-person group, which demonstrates that coaching via telehealth is effective (Ruble et al., 2013).

Overall, the use of telehealth, specifically videoconferencing and coaching, has been found to be a feasible and satisfactory means to deliver a variety to interventions to children and adolescents with ASD. Of the intervention articles included in this review, three studies were directly and/or indirectly impacted by the COVID-19 pandemic in some way (e.g., data collection, social validity questionnaire results) and provide even more support for the use of telehealth as a means to deliver treatment effectively to children with ASD (Cihon et al., 2021; Gerow, et al., 2021a, b; Yi \& Dixon, 2021). Additionally, as indicated earlier, one study focused on an intervention for mask wearing, which is still currently and vitally important (Sivaraman et al., 2021). Parents and teachers were the direct recipient of the telehealth coaching and guidance in 43 out of 47 studies included in the intervention section; this demonstrates that parents and teachers continue to be necessary facilitators of interventions for children with ASD. Lastly, five studies directly compared telehealth interventions to in-person interventions (Baharav \& Reiser, 2010; Kuravackel et al., 2018; Lindgren et al., 2016; Marino et al., 2020; Ruble et al., 2013). Results of four studies yielded no differences between intervention groups in the measured variables (e.g., reduction in problem 
behavior, increase in communication skills) indicating that telehealth may be just as beneficial a treatment medium as in-person services (Baharav \& Reiser, 2010; Kuravackel et al., 2018; Lindgren et al., 2016; Ruble et al., 2013). The study conducted by Marino and colleagues found that parent stress decreased in the telehealth group (had VC coaching) but not the control group, which further supports the use of telehealth as a means to deliver intervention to children with ASD.

\section{Discussion}

The present systematic review sought to explore the literature on telehealth services for individuals with ASD that has been published in the last decade. The 55 telehealth articles examined the types of services and the clinical and implementation outcomes. A range of services were provided via telehealth modalities for assessment (i.e., diagnostic assessments, preference assessments, functional assessments, and speech and language assessments) and treatment (i.e., behavior management, functional communication training, applied behavioral, social communication interventions, and other targeted interventions). The majority of studies (3 assessment and 34 treatment) focused on the parent as the direct recipient of the telehealth intervention. Furthermore, the outcomes from the majority of the studies suggested that the use of telehealth services is possibly equivalent to in-person services or at least superior to control groups. This finding is in line with the previous reviews conducted by Boisvert et al. (2010) and Sutherland et al. (2019).

Despite the last systematic review being relatively recent, there has been a large increase in the number of telehealth research articles produced since publication. The previous systematic review found only 14 articles with eligible studies and an addition 41 studies have emerged that met eligibility. This sharp increase indicates both a shift in the acceptability and implementation of telehealth modalities for individuals diagnosed with ASD as well as a direct response to the consequences of the COVID-19 pandemic. We were able to extend the findings from the previous review in terms of delivery methods and types of services. Since the last review in 2018, the types of intervention and assessment services has grown, which ultimately has increased the need to review and evaluate the literature. Research examining the use of telehealth allows for intervention and assessment services to be provided in equivalence or in conjunction with in-person services.

The findings here are relevant to the implementation of mental health services for individuals with ASD. The majority of the studies included in the review focused on the implementation of intervention services for children and parents, such as communication skills, parent training, cognitive behavioral therapy for anxiety, and ABA. These evidence-based treatments have been the gold standards for in-person interventions. The increase in literature examining the services via telehealth allows for clinicians to target individuals who may not have access to in-person services, such as those suffering from a lack of service providers, or those from lower socioeconomic status or underrepresented racial and ethnic minorities (Pickard et al., 2019). Additionally, with the risk associated with the COVID-19 pandemic, the ability to apply evidence-based treatment services to a telehealth modality is even more essential. Studies that have focused on the implementation of telehealth have continued to find promising results in changes in outcomes for children with ASD as well as the social validity of telehealth (e.g., feasibility, acceptability, satisfaction).

It is important to also consider the access to technology, internet, and/or equipment used across telehealth studies. All of the studies in this review commented on the different telehealth platforms and equipment utilized by both the individual providing the coaching and the participant receiving the telehealth intervention. Twenty-one out of the 55 studies, indicated that they would be able to provide the equipment necessary for the families to participate in their studies (Baharav \& Reiser, 2010; D'Agostino et al., 2020; Fisher et al., 2020; Gerow et al., 2021a, b; Hepburn et al., 2016; Ingersoll et al., 2016; Ingersoll \& Berger, 2015; Lindgren et al., 2016; Machalicek et al., 2016; McCrae et al., 2020; Meadan et al., 2016; O'Brien et al., 2021; Pickard et al., 2016; Schieltz et al., 2018; Simacek et al., 2017; Subramaniam et al., 2017; Suess et al., 2014, 2020; Vismara et al., 2012, 2013). This may have allowed for more families to participate who may not have had access to the technology required. There were ten additional studies that used telehealth centers, clinics, or schools that already had the equipment necessary for participation (Ausenhus \& Higgins, 2019; Corona et al., 2021; Higgins et al., 2017; Machalicek et al., 2009, 2010; Neely et al., 2016, 2018; Suess et al., 2016; Sutherland et al., 2019; Wacker et al., 2013). However, the 24 remaining studies (43\%) either indicated that participants utilized their own technology and equipment, or the study required (as inclusion criterion) access to technology (e.g., desktop computer, laptop, webcam) and internet. This may have limited the number of families who were able to participate in these particular studies; it is advantageous to be able to help provide the necessary technology to be allinclusive in who can participate.

Furthermore, only two studies which focused on the diagnostic assessment of children with ASD via telehealth used participants with a current ASD diagnosis (an inclusion criterion of this review). The initial literature search yielded more than 8 studies exploring the diagnostic assessment of ASD, but upon further examination, these studies primarily used children who were "at risk" for ASD or researchers 
used standardized assessment procedures during the course of the study as corroborating evidence for diagnostic accuracy since the participants were not initially enrolled with a current ASD diagnosis (Juárez et al., 2018; Smith et al., 2017). Interestingly, only one used standardized ASD assessments (i.e., ADOS, ADI-R; Lord et al., 2002) to determine their efficacy in accurately assessing ASD over telehealth (Reese et al., 2013) and one used other measures (e.g., TELE-ASD-PEDS) to accurately diagnosis via telehealth (Corona et al., 2021). More studies need to be conducted examining the efficacy of utilizing standardized ASD assessment measures (e.g., ADOS-2), which are widely used to diagnosis ASD in-person, with participants with established diagnoses to accurately inform their accuracy and use via the telehealth medium. Despite this, the diagnostic studies included in the systematic review provide insight on the ability to remotely deliver important services. Individuals living in rural areas or who are below the poverty line are diagnosed with ASD at much slower rates and at later ages (Antezana et al., 2017). The ability to provide evidencebased services to diagnose ASD via telehealth modalities can potentially reduce the service disparity experienced among this population. Further replication and more studies that examine the use of standardized measures of ASD would further solidify the use of telehealth to accurately diagnosis ASD.

A variety of interventions (e.g., ABA, FCT, social communication) were found to be successfully delivered through videoconferencing platforms and lead to specific childrelated outcomes such as reductions in problem behaviors or increases in social communication skills. The majority of intervention studies utilized free, cost-effective telehealth platforms (e.g., Zoom, Skype) while only one used a specific videoconferencing software at regional clinics and a teleconsultation center (Wacker et al., 2013). The results yielded from this review provides evidence that telehealth can be a medium to deliver pertinent services to children with ASD.

\section{Limitations and Future Directions}

While the current review and state of the literature is encouraging of the use of telehealth in those with ASD, there understandably remain a number of limitations. First, the review does not include unpublished work, which may further benefit the scope of telehealth for individuals with ASD. Although there were two authors reviewing the articles for criteria to ensure reliability, there may be appropriate articles that were missed in the process due to the inclusion criteria that was set. Additionally, due to the COVID-19 pandemic, there has been a recent increase in the use of telehealth services for both intervention and assessment services for individuals with ASD. However, the current review only dates to March 2021 to provide insight on the scope of established telehealth services being implemented prior to the pandemic.

Additional research would be beneficial to explore variations and success rates among providers utilizing technology for services with individuals with ASD during the COVID-19 pandemic, as many of the articles published within the last year did not mention the impact of COVID19 on their study. Only three studies, indicated how they were effected by the COVID-19 pandemic and only one study described the adjustments they made (Yi \& Dixon, 2021).

Additionally, research focusing on providing intervention and assessment services to individuals with ASD and other comorbid disorders is also lacking, there was only one study focusing on comorbid anxiety in children with ASD (Hepburn et al., 2016). Without further exploring the utility of telehealth services in comorbid disorders, these services would not be applicable to many individuals with ASD. Future research should explore the use of telehealth intervention and assessment services with individuals with comorbid disorders. Even though the studies reviewed were largely amenable to implementation via telehealth, it remains to be seen if the full range of ASD presentations can be adequately accommodated and adapted to this modality. Depending on the severity of an individual's presentation, it may also be necessary to consider the safety of individuals on the other end of the platform (e.g., safely addressing challenging behaviors like aggression and self-injury without the safety of trained personnel to handle extinction bursts, etc.). Here, good telehealth practices are important as adequate safety plans and emergency procedures should be created and reviewed in advance. Licensure laws and state regulations also require consideration. It is imperative that practitioners stay current with guidelines involving practicing over great distances and possibly even state/national boundaries. As well, an informed risk vs. benefit calculation must be conducted by both the patient and the practitioner going forward given the frequency with which we see data breaches, $3^{\text {rd }}$ party sharing of information by tech companies, and accidental (and sometimes unfortunately malicious) video "bombing" of uninvited participants joining/disrupting sessions. Finally, while more studies have explored participant satisfaction, cost, and acceptability since the previous review, these factors should continue to be explored to ensure the feasibility of telehealth for the families and individuals with ASD. Along these lines, telehealth interventions should move beyond merely mirroring in-person services (e.g., offering typical manualized CBT for anxiety as practiced in-person). Researchers should investigate specialized techniques and procedures which may yet need to be developed (as opposed to just adaptations of existing treatments) and alterations may be 
desirable to maximize treatment effects specifically via a telehealth platform. As well, every effort should be made to be sure results obtained via telehealth generalize to other "real-world" situations beyond electronic screens.

\section{Conclusion}

Telehealth services for individuals with ASD has begun to emerge as a useful tool for assessment and intervention services relevant to mental health services. With advances in technology and the recent need for telehealth as a result of the COVID-19 pandemic, the literature examining the uses and feasibility of telehealth has greatly increased. The current review suggests there are many uses for telehealth in terms of providing assessment, intervention, and training that benefit individuals or families of individuals with ASD. Modifications to treatment and assessment for individuals with ASD has been found to be successful for in-person services, which may also be beneficial for services provided via technology. Modifications that may be beneficial include using more concrete skills, visual tactics, child-specific interests, and parental involvement (Moree \& Davis, 2010). Further research is needed, however, to determine the success of these modifications via telehealth, as well as any additional uses of telehealth with individuals with ASD.

\section{Declarations}

Conflict of interest The authors have no conflict of interest to declare.

Ethical approval All procedures performed in studies involving human participants were in accordance with the ethical standards of the institutional and/or national research committee and with the $1964 \mathrm{Hel}-$ sinki declaration and its later amendments or comparable ethical standard. Informed consent was obtained from all individual participants included in the study.

\section{References}

American Psychiatric Association. (2013). Diagnostic and statistical manual of mental disorders, fifth edition: DSM-5 (5th ed.). American Psychiatric Association.

Antezana, L., Scarpa, A., Valdespino, A., Albright, J., \& Richey, J. A. (2017). Rural trends in diagnosis and services for autism spectrum disorder. Frontiers in Psychology. https://doi.org/10. 3389/fpsyg.2017.00590

Ausenhus, J. A., \& Higgins, W. J. (2019). An evaluation of realtime feedback delivered via telehealth: Training staff to conduct preference assessments. Behavior Analysis in Practice, 12(3), 643-648. https://doi.org/10.1007/s40617-018-00326-1
Baharav, E., \& Reiser, C. (2010). Using telepractice in parent training in early autism. Telemedicine and e-Health. https://doi.org/ 10.1089/tmj.2010.0029

Barkaia, A., Stokes, T. F., \& Mikiashvili, T. (2017). Intercontinental telehealth coaching of therapists to improve verbalizations by children with autism. Journal of Applied Behavior Analysis, 50(3), 582-589. https://doi.org/10.1002/jaba.391

Baweja, R., Brown, S., Edwards, E., \& Murray, M. (2021). COVID19 pandemic and impact on patients with autism spectrum disorder. Journal of Autism and Developmental Disorders, 10, 1.

Bearss, K., Burrell, T. L., Challa, S. A., Postorino, V., Gillespie, S. E., Crooks, C., \& Scahill, L. (2018). Feasibility of parent training via telehealth for children with autism spectrum disorder and disruptive behavior: A demonstration pilot. Journal of Autism and Developmental Disorders, 48(4), 1020-1030. https://doi.org/10.1007/s10803-017-3363-2

Benson, S. S., Dimian, A. F., Elmquist, M., Simacek, J., McComas, J. J., \& Symons, F. J. (2018). Coaching parents to assess and treat self-injurious behaviour via telehealth. Journal of Intellectual Disability Research, 62(12), 1114-1123. https://doi.org/ 10.1111/jir. 12456

Boisvert, M., Lang, R., Andrianopoulos, M., \& Boscardin, M. L. (2010). Telepractice in the assessment and treatment of individuals with autism spectrum disorders: A systematic review. Developmental Neurorehabilitation, 13(6), 423-432. https://doi.org/10. 3109/17518423.2010.499889.

Camden, C., \& Silva, M. (2021). Pediatric teleheath: Opportunities created by the COVID-19 and suggestions to sustain its use to support families of children with disabilities. Physical \& Occupational Therapy in Pediatrics, 41(1), 1-17. https://doi.org/10. 1080/01942638.2020.1825032

Cihon, J. H., Ferguson, J. L., Lee, M., Leaf, J. B., Leaf, R., \& McEachin, J. (2021). Evaluating the cool versus not cool procedure via telehealth. Behavior Analysis in Practice. https://doi. org/10.1007/s40617-021-00553-z

Corona, L. L., Weitlauf, A. S., Hine, J., Berman, A., Miceli, A., Nicholson, A., Stone, C., Broderick, N., Francis, S., Juárez, A. P., Vehorn, A., Wagner, L., \& Warren, Z. (2021). Parent perceptions of caregiver-mediated telemedicine tools for assessing autism risk in toddlers. Journal of Autism and Developmental Disorders, 51(2), 476-486. https://doi.org/10.1007/ s10803-020-04554-9

D’Agostino, S., Douglas, S. N., \& Horton, E. (2020). Inclusive preschool practitioners' implementation of naturalistic developmental behavioral intervention using telehealth training. Journal of Autism and Developmental Disorders, 50(3), 864-880. https:// doi.org/10.1007/s10803-019-04319-z

Dahiya, A. V., McDonnell, C., DeLucia, E., \& Scarpa, A. (2020). A systematic review of remote telehealth assessments for early signs of autism spectrum disorder: Video and mobile applications. Practice Innovations, 5(2), 150. https://doi.org/10.1037/ pri0000121

Davis, C., Hendon, F., McDonald, K., \& Blanco, S. (2020). Use of technology in facilitating remote caregiver training for token systems. International Journal of Developmental Disabilities, 66(5), 330-338. https://doi.org/10.1080/20473869.2020.1827210

Eshraghi, A. A., Li, C., Alessandri, M., Messinger, D. S., Eshraghi, R. S., Mittal, R., \& Armstrong, F. D. (2020). COVID-19: Overcoming the challenges faced by individuals with autism and their families. The Lancet Psychiatry. https://doi.org/10.1016/S22150366(20)30197-8

Ferguson, J. L., Majeski, M. J., McEachin, J., Leaf, R., Cihon, J. H., \& Leaf, J. B. (2020). Evaluating discrete trial teaching with instructive feedback delivered in a dyad arrangement via telehealth. Journal of Applied Behavior Analysis, 53(4), 1876-1888. https://doi. org/10.1002/jaba.773 
Fisher, W. W., Luczynski, K. C., Blowers, A. P., Vosters, M. E., Pisman, M. D., Craig, A. R., Hood, S. A., Machado, M. A., Lesser, A. D., \& Piazza, C. C. (2020). A randomized clinical trial of a virtual-training program for teaching applied-behavior-analysis skills to parents of children with autism spectrum disorder. Journal of Applied Behavior Analysis, 53(4), 1856-1875. https://doi. org/10.1002/jaba.778

Gerow, S., Radhakrishnan, S., Akers, J., McGinnis, K., \& Swensson, R. (2021a). Telehealth parent coaching to improve daily living skills for children with asd. Journal of Applied Behavior Analysis. https://doi.org/10.1002/jaba.813

Gerow, S., Radhakrishnan, S., Davis, T. N., Zambrano, J., Avery, S., Cosottile, D. W., \& Exline, E. (2021b). Parent-implemented brief functional analysis and treatment with coaching via telehealth. Journal of Applied Behavior Analysis, 54(1), 54-69. https://doi. org/10.1002/jaba.801

Gibson, J. L., Pennington, R. C., Stenhoff, D. M., \& Hopper, J. S. (2010). Using desktop videoconferencing to deliver interventions to a preschool student with autism. Topics in Early Childhood Special Education, 29(4), 214-225. https://doi.org/10. $1177 / 0271121409352873$

Guðmundsdóttir, K., Ala'i-Rosales, S., \& Sigurðardóttir, Z. G. (2019). Extending caregiver training via telecommunication for rural icelandic children with autism. Rural Special Education Quarterly, 38(1), 26-42. https://doi.org/10.1177/8756870518 783522

Guðmundsdóttir, K., Sigurðardóttir, Z. G., \& Ala'i-Rosales, S. (2017). Evaluation of caregiver training via telecommunication for rural Icelandic children with autism. Behavioral Development Bulletin, 22(1), 215-229. https://doi.org/10.1037/bdb00 00040

Hao, Y., Franco, J. H., Sundarrajan, M., \& Chen, Y. (2021). A pilot study comparing tele-therapy and in-person therapy: Perspectives from parent-mediated intervention for children with autism spectrum disorders. Journal of Autism \& Developmental Disorders, 51(1), 129-143.

Hepburn, S. L., Blakeley-Smith, A., Wolff, B., \& Reaven, J. A. (2016). Telehealth delivery of cognitive-behavioral intervention to youth with autism spectrum disorder and anxiety: A pilot study. Autism, 20(2), 207-218. https://doi.org/10.1177/1362361315575164

Higgins, W. J., Luczynski, K. C., Carroll, R. A., Fisher, W. W., \& Mudford, O. C. (2017). Evaluation of a telehealth training package to remotely train staff to conduct a preference assessment. Journal of Applied Behavior Analysis, 50(2), 238-251. https://doi.org/10. 1002/jaba.370

Hyman, S. L., Levy, S. E., Myers, S. M., \& Council on Children with Disabilities, Section on Developmental and Behavioral Pediatrics. (2020). Identification, evaluation, and management of children with autism spectrum disorder. Pediatrics, 145(1), e20193447. https://doi.org/10.1542/peds.2019-3447

Ingersoll, B., \& Berger, N. I. (2015). Parent engagement with a telehealth-based parent-mediated intervention program for children with autism spectrum disorders: Predictors of program use and parent outcomes. Journal of Medical Internet Research, 17(10), e227. https://doi.org/10.2196/jmir.4913

Ingersoll, B., Wainer, A. L., Berger, N. I., Pickard, K. E., \& Bonter, N. (2016). Comparison of a self-directed and therapist-assisted telehealth parent-mediated intervention for children with ASD: A pilot RCT. Journal of Autism and Developmental Disorders, 46(7), 2275-2284. https://doi.org/10.1007/s10803-016-2755-z

Juárez, A. P., Weitlauf, A. S., Nicholson, A., Pasternak, A., Broderick, N., Hine, J., Stainbrook, J. A., \& Warren, Z. (2018). Early identification of ASD through telemedicine: Potential value for underserved populations. Journal of Autism and Developmental Disorders, 48(8), 2601-2610. https://doi.org/10.1007/ s10803-018-3524-y
Kuravackel, G. M., Ruble, L. A., Reese, R. J., Ables, A. P., Rodgers, A. D., \& Toland, M. D. (2018). COMPASS for hope: Evaluating the effectiveness of a parent training and support program for children with ASD. Journal of Autism and Developmental Disorders, 48(2), 404-416. https://doi.org/10.1007/s10803-017-3333-8

Lindgren, S., Wacker, D., Schieltz, K., Suess, A., Pelzel, K., Kopelman, T., Lee, J., Romani, P., \& O’Brien, M. (2020). A randomized controlled trial of functional communication training via telehealth for young children with autism spectrum disorder. Journal of Autism and Developmental Disorders. https://doi.org/10.1007/ s10803-020-04451-1

Lindgren, S., Wacker, D., Suess, A., Schieltz, K., Pelzel, K., Kopelman, T., Lee, J., Romani, P., \& Waldron, D. (2016). Telehealth and autism: Treating challenging behavior at lower cost. Pediatrics, 137(Supplement), S167-S175. https://doi.org/10.1542/peds. 2015-2851O

Lord, C., Rutter, M., DiLavore, P. C., Risi, S., \& Somer, L. (2002). Autism diagnostic observation schedule. Western Psychological Services.

Luxton, D. D., Nelson, E.-L., \& Maheu, M. M. (2016). A practitioner's guide to telemental health: How to conduct legal, ethical, and evidence-based telepractice. American Psychological Association. https://doi.org/10.1037/14938-000

Machalicek, W., Lequia, J., Pinkelman, S., Knowles, C., Raulston, T., Davis, T., \& Alresheed, F. (2016). Behavioral telehealth consultation with families of children with autism spectrum disorder. Behavioral Interventions, 31(3), 223-250. https://doi.org/10.1002/ bin. 1450

Machalicek, W., O’Reilly, M., Chan, J. M., Rispoli, M., Lang, R., Davis, T., Shogren, K., Sorrells, A., Lancioni, G., Sigafoos, J., Green, V., \& Langthorne, P. (2009). Using videoconferencing to support teachers to conduct preference assessments with students with autism and developmental disabilities. Research in Autism Spectrum Disorders, 3(1), 32-41. https://doi.org/10.1016/j.rasd. 2008.03.004

Machalicek, W., O’Reilly, M. F., Rispoli, M., Davis, T., Lang, R., Franco, J. H., \& Chan, J. M. (2010). Training teachers to assess the challenging behaviors of students with autism using video tele-conferencing. Education and Training in Autism and Developmental Disabilities, 45, 203-215.

Marino, F., Chilà, P., Failla, C., Crimi, I., Minutoli, R., Puglisi, A., Arnao, A. A., Tartarisco, G., Ruta, L., Vagni, D., \& Pioggia, G. (2020). Tele-assisted behavioral intervention for families with children with autism spectrum disorders: A randomized control trial. Brain Sciences, 10(9), 649. https://doi.org/10.3390/brain sci10090649

McCrae, C. S., Chan, W. S., Curtis, A. F., Nair, N., Deroche, C. B., Munoz, M., Takamatsu, S., McLean, D., Davenport, M., Muckerman, J. E., Takahashi, N., McCann, D., McGovney, K., Sahota, P., \& Mazurek, M. O. (2020). Telehealth cognitive behavioral therapy for insomnia in children with autism spectrum disorder: A pilot examining feasibility, satisfaction, and preliminary findings. Autism: the International Journal of Research and Practice. https://doi.org/10.1177/1362361320949078

Meadan, H., Snodgrass, M. R., Meyer, L. E., Fisher, K. W., Chung, M. Y., \& Halle, J. W. (2016). Internet-based parent-implemented intervention for young children with autism: A pilot study. Journal of Early Intervention, 38(1), 3-23. https://doi.org/10.1177/10538 15116630327

Moree, B. N., \& Davis, T. E. (2010). Cognitive-behavioral therapy for anxiety in children diagnosed with autism spectrum disorders: Modification trends. Research in Autism Spectrum Disorders, 4(3), 346-354. https://doi.org/10.1016/j.rasd.2009.10.015

National Autism Center. (2015). Findings and conclusions: National standards project, phase 2. Author Randolph. 
Neely, L., Rispoli, M., Boles, M., Morin, K., Gregori, E., Ninci, J., \& Hagan-Burke, S. (2018). Interventionist acquisition of incidental teaching using pyramidal training via telehealth. Behavior Modification, 43(5), 711-733. https://doi.org/10.1177/0145445518 781770

Neely, L., Rispoli, M., Gerow, S., \& Hong, E. R. (2016). Preparing interventionists via telepractice in incidental teaching for children with autism. Journal of Behavioral Education, 25(4), 393-416. https://doi.org/10.1007/s10864-016-9250-7

O’Brien, M. J., Schieltz, K. M., Berg, W. K., McComas, J. J., \& Wacker, D. P. (2021). Delivering interventions via telehealth: Functional communication training with a child with autism as a case example. Research \& Practice for Persons with Severe Disabilities, 46(1), 53-60.

Pickard, K., Reyes, N., \& Reaven, J. (2019). Examining the inclusion of diverse participants in cognitive behavior therapy research for youth with autism spectrum disorder and anxiety. Autism, 23(4), 1057-1064. https://doi.org/10.1177/1362361318795678

Pickard, K., Wainer, A. L., Bailey, K. M., \& Ingersoll, B. R. (2016). A mixed-method evaluation of the feasibility and acceptability of a telehealth-based parent-mediated intervention for children with autism spectrum disorder. Autism, 20(7), 845-855. https://doi.org/ $10.1177 / 1362361315614496$

Pierson, L. M., Thompson, J. L., Ganz, J. B., Wattanawongwan, S., Haas, A. N., \& Yllades, V. (2021). Coaching parents of children with developmental disabilities to implement a modified dialogic reading intervention using low technology via telepractice. American Journal of Speech-Language Pathology, 30(1), 119-136.

Reaven, J., Blakeley-Smith, A., Nichols, S., \& Hepburn, S. (2011). Facing your fears: Group therapy for managing anxiety in children with high-functioning autism spectrum disorders. Brookes.

Reese, R. M., Jamison, R., Wendland, M., Fleming, K., Braun, M. J., Schuttler, J. O., \& Turek, J. (2013). Evaluating interactive videoconferencing for assessing symptoms of autism. Telemedicine and E-Health, 19(9), 671-677. https://doi.org/10.1089/tmj.2012.0312

Rooks-Ellis, S. K., Howorth, M. K., Boulette, S., \& Sulinski, E. (2020). Effects of a parent training using telehealth: Equity and access to early intervention for rural families. Journal of Childhood, Education \& Society, 1(2), 141-166. https://doi.org/10.37291/27176 38X.20201242

Ruble, L. A., McGrew, J. H., Toland, M. D., Dalrymple, N. J., \& Jung, L. A. (2013). A randomized controlled trial of COMPASS webbased and face-to-face teacher coaching in autism. Journal of Consulting and Clinical Psychology, 81(3), 566-572. https://doi. org/10.1037/a0032003

Rutter, M., Le Couteur, A., \& Lord, C. (2003). Autism diagnostic interview-revised. Western Psychological Services.

Schieltz, K. M., Romani, P. W., Wacker, D. P., Suess, A. N., Huang, P., Berg, W. K., Lindgren, S. D., \& Kopelman, T. G. (2018). Single-case analysis to determine reasons for failure of behavioral treatment via telehealth. Remedial and Special Education, 39(2), 95-105. https://doi.org/10.1177/0741932517743791

Schlosser, R. W., Shane, H. C., Allen, A. A., Benz, A., Cullen, J., O’Neill, L., Chiesa, L., Miori-Dinneen, L., Koul, R., \& Pasupathy, R. (2020). Coaching a school team to implement the visual immersion system ${ }^{\mathrm{TM}}$ in a classroom for children with autism spectrum disorder: A mixed-methods proof-of-concept study. Advances in Neurodevelopmental Disorders: Multidisciplinary Research and
Practice across the Lifespan, 4(4), 447. https://doi.org/10.1007/ s41252-020-00176-5

Shulver, W., Killington, M., \& Crotty, M. (2016). 'Massive potential' or 'safety risk'? Health worker views on telehealth in the care of older people and implications for successful normalization. BMC Medical Informatics and Decision Making, 16(1), 131. https://doi. org/10.1186/s12911-016-0373-5

Simacek, J., Dimian, A. F., \& McComas, J. J. (2017). Communication intervention for young children with severe neurodevelopmental disabilities via telehealth. Journal of Autism and Developmental Disorders, 47(3), 744-767. https://doi.org/10.1007/ s10803-016-3006-z

Singh, N. N., Lancioni, G. E., Medvedev, O. N., Hwang, Y.-S., \& Myers, R. E. (2021). Real-time telehealth treatment team consultation for self-injury by individuals with autism spectrum disorder. Advances in Neurodevelopmental Disorders. https://doi.org/10. 1007/s41252-021-00192-z

Sivaraman, M., Virues-Ortega, J., \& Roeyers, H. (2021). Telehealth mask wearing training for children with autism during the COVID-19 pandemic. Journal of Applied Behavior Analysis, 54(1), 70-86. https://doi.org/10.1002/jaba.802

Smith, C. J., Rozga, A., Matthews, N., Oberleitner, R., Nazneen, N., \& Abowd, G. (2017). Investigating the accuracy of a novel telehealth diagnostic approach for autism spectrum disorder. Psychological Assessment, 29(3), 245-252. https://doi.org/10.1037/pas0000317

Stone, W. L., Coonrod, E. E., \& Ousley, O. Y. (2000). Brief report: Screening tool for autism in two-year-olds (STAT): Development and preliminary data. Journal of Autism and Developmental Disorders, 30(6), 607-612. https://doi.org/10.1023/A:1005647629 002

Subramaniam, S., Brunson, L. Y., Cook, J. E., Larson, N. A., Poe, S. G., \& St Peter, C. C. (2017). Maintenance of parent-implemented discrete-trial instruction during Videoconferencing. Journal of Behavioral Education, 26(1), 1-26. https://doi.org/10.1007/ s10864-016-9258-z

Suess, A. N., Romani, P. W., Wacker, D. P., Dyson, S. M., Kuhle, J. L., Lee, J. F., Lindgren, S. D., Kopelman, T. G., Pelzel, K. E., \& Waldron, D. B. (2014). Evaluating the treatment fidelity of parents who conduct in-home functional communication training with coaching via telehealth. Journal of Behavioral Education, 23(1), 34-59. https://doi.org/10.1007/s10864-013-9183-3

Suess, A. N., Schieltz, K. M., Wacker, D. P., Detrick, J., \& Podlesnik, C. A. (2020). An evaluation of resurgence following functional communication training conducted in alternative antecedent contexts via telehealth. Journal of the Experimental Analysis of Behavior, 113(1), 278-301. https://doi.org/10.1002/jeab.551

Suess, A. N., Wacker, D. P., Schwartz, J. E., Lustig, N., \& Detrick, J. (2016). Preliminary evidence on the use of telehealth in an outpatient behavior clinic. Journal of Applied Behavior Analysis, 49(3), 686-692. https://doi.org/10.1002/jaba.305

Sutherland, R., Trembath, D., Hodge, M. A., Rose, V., \& Roberts, J. (2019). Telehealth and autism: Are telehealth language assessments reliable and feasible for children with autism? International Journal of Language \& Communication Disorders, 54(2), 281291. https://doi.org/10.1111/1460-6984.12440

Vismara, L. A., McCormick, C. E. B., Wagner, A. L., Monlux, K., Nadhan, A., \& Young, G. S. (2018). Telehealth parent training in the early start denver model: Results from a randomized controlled 
study. Focus on Autism and Other Developmental Disabilities, 33(2), 67-79. https://doi.org/10.1177/1088357616651064

Vismara, L. A., McCormick, C., Young, G. S., Nadhan, A., \& Monlux, K. (2013). Preliminary findings of a telehealth approach to parent training in autism. Journal of Autism and Developmental Disorders, 43(12), 2953-2969. https://doi.org/10.1007/ s10803-013-1841-8

Vismara, L. A., Young, G. S., \& Rogers, S. J. (2012). Telehealth for expanding the reach of early autism training to parents. Autism Research and Treatment, 2012, 1-12. https://doi.org/10.1155/ 2012/121878

Wacker, D. P., Lee, J. F., Padilla Dalmau, Y. C., Kopelman, T. G., Lindgren, S. D., Kuhle, J., Pelzel, K. E., Dyson, S., Schieltz, K. M., \& Waldron, D. B. (2013). Conducting functional communication training via telehealth to reduce the problem behavior of young children with autism. Journal of Developmental and
Physical Disabilities, 25(1), 35-48. https://doi.org/10.1007/ s10882-012-9314-0

Wainer, A. L., \& Ingersoll, B. R. (2015). Increasing access to an ASD imitation intervention via a telehealth parent training program. Journal of Autism and Developmental Disorders, 45(12), 38773890. https://doi.org/10.1007/s10803-014-2186-7

Yi, Z., \& Dixon, M. R. (2021). Developing and enhancing adherence to a telehealth ABA parent training curriculum for caregivers of children with autism. Behavior Analysis in Practice (springer Science \& Business Media BV), 14(1), 58-74.

Publisher's Note Springer Nature remains neutral with regard to jurisdictional claims in published maps and institutional affiliations. 